\title{
STABILITY AND CONVERGENCE OF TWO DISCRETE SCHEMES FOR A DEGENERATE SOLUTAL NON-ISOTHERMAL PHASE-FIELD MODEL*
}

\author{
Francisco Guillén-González ${ }^{1}$ and Juan Vicente Gutiérrez-Santacred ${ }^{1}$
}

\begin{abstract}
We analyze two numerical schemes of Euler type in time and $C^{0}$ finite-element type with $\mathbb{P}_{1}$-approximation in space for solving a phase-field model of a binary alloy with thermal properties. This model is written as a highly non-linear parabolic system with three unknowns: phase-field, solute concentration and temperature, where the diffusion for the temperature and solute concentration may degenerate. The first scheme is nonlinear, unconditionally stable and convergent. The other scheme is linear but conditionally stable and convergent. A maximum principle is avoided in both schemes, using a truncation operator on the $L^{2}$ projection onto the $\mathbb{P}_{0}$ finite element for the discrete concentration. In addition, for the model when the heat conductivity and solute diffusion coefficients are constants, optimal error estimates for both schemes are shown based on stability estimates.
\end{abstract}

Mathematics Subject Classification. 35Q72, 35K65,65M12, 65M60.

Received November 15, 2007. Revised May 21, 2008 and October 1st, 2008.

Published online April 30, 2009.

\section{INTRODUCTION}

\subsection{The model}

The phase-field method provides a mathematical description for free-boundary problems associated to physical processes with phase transitions. It postulates the existence of a function, called the phase-field, whose value identifies the phase at a particular point in space and time. The method is particularly suitable for cases with complex growth structures occurring during phase transitions. The mathematical model studied in this work describes the solidification process occurring in a binary alloy with temperature-dependent properties. It is based on a highly non-linear parabolic system of partial differential equations with three dependent variables: phase-field, solute concentration and temperature. Moreover, the temperature and concentration equation have nonlinear degenerate diffusivity.

Keywords and phrases. Phase-field models, diffuse interface model, solidification process, degenerate parabolic systems, backward Euler schemes, finite elements, stability, convergence, error estimates.

* This work has been partially supported by DGI-MEC (Spain), Grant MTM2006-07932 and CGCI MECD-DGU Brazil/Spain, Grant 117/06.

${ }^{1}$ Dpto. E.D.A.N., University of Sevilla, Aptdo. 1160, 41080 Sevilla, Spain. guillen@us.es; juanvi@us.es 
Let $\Omega \subseteq \mathbb{R}^{d}(d=2$ or 3$)$ be a bounded domain with boundary $\Gamma$. Denote by $[0, T]$ the time interval $(T>0)$. We use the notation $Q=\Omega \times(0, T), \Sigma=\Gamma \times(0, T)$ and $\boldsymbol{n}(\boldsymbol{x})$ is the outwards unit normal vector to $\Omega$ at the point $\boldsymbol{x} \in \Gamma$.

After some physical simplifications [5], we consider the following differential problem, related to a phase-field model of a binary alloy with thermal properties [1]:

$$
\left\{\begin{aligned}
\alpha \varepsilon^{2} \phi_{t}-\varepsilon^{2} \Delta \phi & =\frac{1}{2}\left(\phi-\phi^{3}\right)+\beta\left(\theta-\theta_{A} c-\theta_{B}(1-c)\right) & & \text { in } Q \\
C_{V} \theta_{t}+\frac{l}{2} \phi_{t} & =\nabla \cdot\left[K_{1}(\phi) \nabla \theta\right] & & \text { in } Q \\
c_{t} & =\nabla \cdot\left[K_{2}(\phi)(\nabla c+M c(1-c) \nabla \phi)\right] & & \text { in } Q .
\end{aligned}\right.
$$

This model is completed with the Neumann boundary conditions

$$
\left.\frac{\partial \phi}{\partial \boldsymbol{n}}\right|_{\Sigma}=0,\left.\quad\left(K_{1}(\phi) \nabla \theta\right) \cdot \boldsymbol{n}\right|_{\Sigma}=0,\left.\quad\left(K_{2}(\phi) \nabla c\right) \cdot \boldsymbol{n}\right|_{\Sigma}=0
$$

and the initial conditions

$$
\phi(\boldsymbol{x}, 0)=\phi_{0}(\boldsymbol{x}), \quad \theta(\boldsymbol{x}, 0)=\theta_{0}(\boldsymbol{x}), \quad c(\boldsymbol{x}, 0)=c_{0}(\boldsymbol{x}) \quad \boldsymbol{x} \in \Omega .
$$

The unknowns for this problem are: $\phi: Q \rightarrow \mathbb{R}$ (phase-field) is the state variable characterizing the different phases so that $\phi=1$ represents the liquid phase and $\phi=-1$ represents the solid phase, $\theta: Q \rightarrow \mathbb{R}$ is the temperature of the material, $c: Q \rightarrow[0,1]$ (concentration) represents the fraction of one of the two materials in the mixture. The parameter $\alpha>0$ is the relaxation scaling; the parameter $\beta$ is given by $\beta=\varepsilon[s] / 3 \sigma$, where $\varepsilon>0$ is the measure of the interface width, $\sigma$ the surface tension and $[s]$ the entropy density difference between phases; $\theta_{A}, \theta_{B}$ are the melting temperatures of each of the two materials in the alloy; $C_{V}>0$ is the specific heat; $l>0$ the latent heat; $K_{1} \geq 0$ the thermal conductivity; $K_{2} \geq 0$ the solute diffusivity; $M \in \mathbb{R}$ is a constant related to the slopes of solid and liquid lines.

We will assume that $K_{1}=K_{1}(\phi)$ and $K_{2}=K_{2}(\phi)$ are two globally Lipschitz continuous functions satisfying

$$
0 \leq K_{1}(r) \leq b_{1}, \quad 0 \leq K_{2}(r) \leq b_{2} \quad \forall r \in \mathbb{R}
$$

with $b_{1}, b_{2}>0$. In this sense, the problem is singular with respect to the temperature and concentration when $K_{1}(\phi)=0$ or $K_{2}(\phi)=0$, respectively. As physically the diffusion of material in the solid phase can be considered close to zero [5]; this leads to a degenerate solute diffusion. Such a phenomenon is included in this model, assuming that $K_{2}(\phi)=0$ if $\phi=-1$. On the other hand, although the heat conductivity is nonzero in both solid and liquid phases, we also consider a degenerate diffusion for the temperature with the aim of considering a more general model. Moreover, this may help the development of numerical methods for systems with similar characteristics.

The phase-field model for solidification (1.1) is used to treat phenomena such as crystal growth and the fusion of materials.

Now we introduce the definition of weak solutions similar to that given in $[1,12]$ which take into account the heat and solute degenerate diffusivity, respectively. Moreover, the maximum principle for the concentration equation says us that $0 \leq c \leq 1$ in $Q$ if $0 \leq c_{0} \leq 1$ in $\Omega$.

Definition 1.1. A triplet $(\phi, \theta, c)$ is called a weak solution of $(1.1)-(1.3)$ in $(0, T)$ if:

(1) $\phi \in L^{2}\left(0, T ; H^{2}(\Omega)\right) \cap L^{\infty}\left(0, T, H^{1}(\Omega)\right), \phi_{t} \in L^{2}(Q), \phi(0)=\phi_{0}, \frac{\partial \phi}{\partial \boldsymbol{n}}=0$ a.e. on $\Sigma$,

(2) $\theta \in L^{\infty}\left(0, T ; L^{2}(\Omega)\right), \theta_{t} \in L^{2}\left(0, T, H^{1}(\Omega)^{\prime}\right), \theta(0)=\theta_{0}$,

(3) $c \in L^{\infty}\left(0, T ; L^{2}(\Omega)\right), c_{t} \in L^{2}\left(0, T ; H^{1}(\Omega)^{\prime}\right), c(0)=c_{0}, 0 \leq c \leq 1$ a.e. in $Q$,

(4) $J_{1}:=\nabla\left(K_{1}(\phi) \theta\right)-\theta \nabla K_{1}(\phi) \in L^{2}(Q)$,

(5) $J_{2}:=\nabla\left(K_{2}(\phi) c\right)-c \nabla K_{2}(\phi) \in L^{2}(Q)$, 
verifying

$$
\begin{gathered}
\alpha \varepsilon^{2} \phi_{t}-\varepsilon^{2} \Delta \phi=\frac{1}{2}\left(\phi-\phi^{3}\right)+\beta\left(\theta-\theta_{A} c-\theta_{B}(1-c)\right) \quad \text { a.e. in } Q, \\
C_{V} \int_{0}^{T}\left\langle\theta_{t}, \eta\right\rangle \mathrm{d} t+\frac{l}{2} \int_{0}^{T}\left(\phi_{t}, \eta\right) \mathrm{d} t+\int_{0}^{T}\left(J_{1}, \nabla \eta\right)=0, \\
\int_{0}^{T}\left\langle c_{t}, \eta\right\rangle \mathrm{d} t+\int_{0}^{T}\left(J_{2}, \nabla \eta\right) \mathrm{d} t+M \int_{0}^{T}\left(K_{2}(\phi) c(1-c) \nabla \phi, \nabla \eta\right)=0,
\end{gathered}
$$

for each $\eta \in L^{2}\left(0, T ; H^{1}(\Omega)\right)$. If, in addition, $K_{1}, K_{2} \geq b_{0}>0$, then $\theta, c \in L^{2}\left(0, T ; H^{1}(\Omega)\right)$ and $J_{1}=K_{1}(\phi) \nabla \theta$, $J_{2}=K_{1}(\phi) \nabla c$.

Here and in what follows, $(\cdot, \cdot)$ denotes the inner product in $L^{2}(\Omega)$ and $\langle\cdot, \cdot\rangle$ denotes the duality between $H^{1}(\Omega)^{\prime}$ and $H^{1}(\Omega)$.

\subsection{Known results}

In [1], the existence of weak solutions of problem (1.1)-(1.3) but with a constant solute diffusivity $\left(K_{2}>0\right)$ is obtained via the introduction of a regularized problem approximating the degenerate thermal conductivity $K_{1}$ by a strictly positive, regular function followed by the derivation of suitable a priori estimates and the application of compactness arguments. More concretely, the following existence result was established in [1].

Theorem 1.2. Let $\Omega$ be an open bounded domain of $\mathbb{R}^{d}, d=2$ or 3 , with smooth boundary $\Gamma$. Assume $\phi_{0} \in H^{1+\gamma}(\Omega)$ with $1 / 2<\gamma \leq 1$ such that $\frac{\partial \phi_{0}}{\partial \mathbf{n}}=0$ on $\Gamma, \theta_{0} \in L^{2}(\Omega)$ and $c_{0} \in H^{1}(\Omega)$ such that $0 \leq c_{0} \leq 1$ a.e. in $\bar{\Omega}$. Then, there exists $(\phi, \theta, c)$ a weak solution of (1.1)-(1.3) (with $K_{1}>0$ a constant) in $(0, T)$.

In addition, in [1] the authors say that the hypothesis $\phi_{0} \in H^{1+\gamma}(\Omega)$ with $1 / 2<\gamma \leq 1$ is not essential, and the result holds for $\phi_{0} \in H^{1}(\Omega)$.

Scheid [12] proved the existence of weak solutions, by using a similar methodology to [1], for the following isothermal phase-field model of a binary alloy

$$
\left\{\begin{aligned}
\alpha \varepsilon^{2} \phi_{t}-\varepsilon^{2} \Delta \phi & =F_{1}(\phi)+c F_{2}(\phi) & & \text { in } Q \\
c_{t} & =\nabla \cdot\left[D_{1}(\phi)\left(\nabla c+D_{2}(c, \phi) \nabla \phi\right)\right] & & \text { in } Q
\end{aligned}\right.
$$

which has a degenerate solute diffusivity $D_{1}(\phi) \geq 0$. The main difficulty of model (1.4) is the treatment of the nonlinear term involving $D_{1}(\phi) D_{2}(c, \phi) \nabla \phi$ in the concentration equation. Moreover, the maximum principle for the phase-field variable gives $-1 \leq \phi \leq 1$ under the assumptions that the above nonlinearities $F_{1}(\phi)$ and $F_{2}(\phi)$ vanish when $\phi=-1$ and $\phi=1$.

Error estimates of nonlinear numerical schemes for isothermal phase-field models related to binary alloys are given in [10] for a model as (1.4), and, in [4], considering anisotropic diffusion for the phase-field equation and a more general right-hand side in the phase-field equation, changing the terms $F_{1}(\phi)+c F_{2}(\phi)$ considered in [10] by $S(c, \phi)$ being a bounded, Lipschitz function.

In [7], optimal error estimates are given for a fully discrete nonlinear numerical scheme of a more simplified phase-field model than (1.1) without the concentration (that is one material is only considered) and with constant thermal conductivity $K_{1}>0$, paying special attention on the dependency of the parameter $\varepsilon$. Stability estimates independent of $\varepsilon$ are proved for $k$ small enough with respect to $\varepsilon$, and $\alpha, \beta$ are constants depending on $\varepsilon$. It is also shown some error bounds depending only on a lower polynomial order for $1 / \varepsilon$. Moreover, error estimates are used to establish the convergence of the fully discrete scheme to solutions of the sharp interface limits under different scaling hypotheses in its coefficients.

In [2], a time-discrete nonlinear scheme is proposed for a phase-field problem again without the concentration variable and replacing in the equation for the temperature the term $\frac{l}{2} \phi_{t}$ by the more general term $\frac{l}{2} f(\theta, \phi)_{t}$, where $f$ is a generic function satisfying some adequate properties. Convergence of this semi-discrete in time scheme is proved, obtaining the existence and regularity of solutions for the limit problem. 


\subsection{Main results of the paper}

In this work we will consider two numerical schemes in order to approximate problem (1.1) using continuous $\mathbb{P}_{1}$-finite elements for the tree variables $(\phi, \theta, c)$. Since a maximum principle cannot be verified in general by the discrete concentration, we introduce a truncation operator on the $L^{2}$ projection onto $\mathbb{P}_{0}$, in order to guarantee a $L^{\infty}$ bound for some terms in the discrete concentration equation. A similar idea of truncation, but without the $L^{2}$ projection onto $\mathbb{P}_{0}$, has been used in [8] for a $2 D$ Navier-Stokes model with mass diffusion.

First of all, we will present in Section 2 the nonlinear numerical scheme (2.1)-(2.3) which will be unconditionally stable and convergent.

Theorem 1.3 (unconditionally stable, convergent nonlinear scheme). Assume

$$
\phi_{0} \in H^{1}(\Omega), \quad \theta_{0} \in L^{2}(\Omega) \quad \text { and } \quad c_{0} \in L^{2}(\Omega) \quad \text { such that } 0 \leq c_{0} \leq 1 \quad \text { a.e. in } \bar{\Omega} .
$$

Let $\Omega$ be such that the $H^{2}$-regularity for the Neumann problem (3.19) holds. Let $\mathcal{T}_{h}$ be a regular, quasi-uniform family of a polyhedral domain $\Omega$. Then, there exists a convergent subsequence of functions $\phi_{h, k}, \theta_{h, k}$ and $c_{h, k}$ associated to scheme (2.1)-(2.3) (see Def. 3.5) towards a weak solution $(\phi, \theta, c)$ of problem $(1.1)-(1.3)$ in $(0, T)$, as $(h, k) \rightarrow 0$ in the following sense:

$$
\begin{gathered}
\theta_{h, k} \rightarrow \theta, \quad c_{h, k} \rightarrow c, \quad \text { in } L^{\infty}\left(0, T ; L^{2}(\Omega)\right) \text {-weak*, } \\
\phi_{h, k} \rightarrow \phi, \quad \text { in } L^{\infty}\left(0, T ; H^{1}(\Omega)\right) \text {-weak*, and in } L^{2}\left(0, T ; H^{1}(\Omega)\right) \text {-strong. }
\end{gathered}
$$

Second, we construct the linear numerical scheme (6.1)-(6.3) which will be conditionally stable and convergent.

Theorem 1.4 (conditionally stable, convergent linear scheme). Assume the assumptions of Theorem 1.3 and the constraint

$$
\lim _{(h, k) \rightarrow 0} \frac{k}{h}=0
$$

Then, there exists a convergent subsequence of functions $\phi_{h, k}, \theta_{h, k}$ and $c_{h, k}$ associated to scheme (6.1)-(6.3) (see Def. 3.5) towards a weak solution $(\phi, \theta, c)$ of problem $(1.1)-(1.3)$ in $(0, T)$, as $(h, k) \rightarrow 0$ in the same sense of Theorem 1.3.

At this point, it is well to point out that, in particular, the two previous theorems provide the existence of weak solutions of problem (1.1)-(1.3) under hypotheses on the data weaker than those imposed in [1] (see Thm. 1.2). To be more precise, the hypothesis on $c_{0}$ is relaxed from $c_{0} \in H^{1}(\Omega)$ imposed in $[1]$ to $c_{0} \in L^{2}(\Omega)$ as was considered in [12]. Recall that in [12] the isothermal case is considered and in [1] there is not degeneration in the solute diffusivity.

Finally, assuming both the heat conductivity and solute diffusion coefficients are constants, error estimates of order $O(k+h)$ are shown towards a regular enough continuous solution to (1.1)-(1.3).

Theorem 1.5. Under hypotheses of Theorem 1.3 (respectively Thm. 1.4), admitting that $K_{1}, K_{2}$ are two positive constants and that there exist a continuous solution $(\phi, \theta, c)$ to $(1.1)-(1.3)$ which verifies the regularity assumptions (7.2), then the discrete solution of scheme (2.1)-(2.3) (respectively (6.1)-(6.3)) satisfies for all $n<N$

$$
\begin{aligned}
\left\|e_{\phi}^{n+1}\right\|_{H^{1}(\Omega)}^{2}+\frac{1}{4 \varepsilon^{2}}\left\|e_{\phi}^{n+1}\right\|_{L^{4}(\Omega)}^{4}+C_{V}\left|e_{\theta}^{n+1}\right|^{2}+\left|e_{c}^{n+1}\right|^{2}+\alpha k \sum_{l=1}^{n}\left|\frac{e_{\phi}^{l+1}-e_{\phi}^{l}}{k}\right|^{2} & \\
& +K_{1} k \sum_{l=1}^{n}\left|\nabla e_{\theta}^{l+1}\right|^{2}+K_{2} k \sum_{l=1}^{n}\left|\nabla e_{c}^{l+1}\right|^{2} \leq C\left(h^{2}+k^{2}\right),
\end{aligned}
$$


where $C$ is a constant independent of $h$ and $k$, and the errors are denoted by $e_{\phi}^{n+1}=\phi_{h}^{n+1}-\phi\left(t_{n+1}\right)$, $e_{\theta}^{n+1}=\theta_{h}^{n+1}-\theta\left(t_{n+1}\right)$ and $e_{c}^{n+1}=c_{h}^{n+1}-c\left(t_{n+1}\right)$.

The rest of the paper is described as follows. In Section 2 the nonlinear scheme (2.1)-(2.3) is presented, obtaining its unconditionally stability in Section 3. In Section 4 some necessary compactness results are proved, passing to the limit in Section 5 and concluding the proof of Theorem 1.3. In addition, a conditionally stable and convergent linear scheme is studied in Section 6 giving an outline of the proof of Theorem 1.4. Finally, Section 7 is devoted to studying optimal error estimates for both schemes.

\section{A NONLINEAR SCHEME}

In what follows, let us consider a uniform partition $t_{n}=n k$ of the time interval $[0, T]$ with $k=T / N$ the time step, let $\Omega \subset \mathbb{R}^{d}\left(d=2\right.$ or 3 ) be a domain with polyhedral boundary and $\mathcal{T}_{h}$ be a family of triangulations of $\Omega$ with $\bar{\Omega}=\bigcup_{K \in \mathcal{T}_{h}} K$. Here $h:=\max _{K \in \mathcal{T}_{h}} h_{K}$ with $h_{K}$ the diameter of $K$. Let $X_{h}$ be the finite element subspace of $H^{1}(\Omega)$ furnished by globally continuous, piecewise linear functions, that is,

$$
X_{h}=\left\{x_{h} \in C^{0}(\bar{\Omega}):\left.x_{h}\right|_{K} \in \mathbb{P}_{1}(K), \quad \forall K \in \mathcal{T}_{h},\right\} .
$$

A first idea to approximate equation $(1.1)_{1}$ is

$$
\left\{\begin{array}{l}
\alpha\left(\frac{\phi_{h}^{n+1}-\phi_{h}^{n}}{k}, x_{h}\right)+\left(\nabla \phi_{h}^{n+1}, \nabla x_{h}\right)+\frac{1}{2 \varepsilon^{2}}\left(\left(\phi_{h}^{n+1}\right)^{3}, x_{h}\right) \\
=\frac{1}{2 \varepsilon^{2}}\left(\phi_{h}^{n}, x_{h}\right)+\frac{\beta}{\varepsilon^{2}}\left(\theta_{h}^{n}-\theta_{A} c_{h}^{n}-\theta_{B}\left(1-c_{h}^{n}\right), x_{h}\right), \quad \forall x_{h} \in X_{h} .
\end{array}\right.
$$

But, we add $\left(\phi_{h}^{n+1}, x_{h}\right)$ to the left-hand side and $\left(\phi_{h}^{n}, x_{h}\right)$ to the right-hand side which will cancel each other in the limit as $(h, k)$ go to zero. The reason why we introduce these terms is to get stability constants only of polynomial order with respect to $\varepsilon$ avoiding exponential dependence. Concretely, since $\beta=O(\varepsilon)$, we will get stability constants depending on $1 / \varepsilon$ (see Rem. 3.2 below).

Then we propose the following scheme to approximate problem (1.1)-(1.3):

Initialization: Let $\left(\phi_{h}^{0}, \theta_{h}^{0}, c_{h}^{0}\right) \in X_{h} \times X_{h} \times X_{h}$ be suitable approximations of $\left(\phi_{0}, \theta_{0}, c_{0}\right)$.

Step $n+1$ : Given $\left(\phi_{h}^{n}, \theta_{h}^{n}, c_{h}^{n}\right) \in X_{h} \times X_{h} \times X_{h}$.

Find $\phi_{h}^{n+1} \in X_{h}$ as a solution of the problem:

$$
\left\{\begin{array}{l}
\alpha\left(\frac{\phi_{h}^{n+1}-\phi_{h}^{n}}{k}, x_{h}\right)+\left(\nabla \phi_{h}^{n+1}, \nabla x_{h}\right)+\left(\phi_{h}^{n+1}, x_{h}\right)+\frac{1}{2 \varepsilon^{2}}\left(\left(\phi_{h}^{n+1}\right)^{3}, x_{h}\right) \\
=\left(\frac{1}{2 \varepsilon^{2}}+1\right)\left(\phi_{h}^{n}, x_{h}\right)+\frac{\beta}{\varepsilon^{2}}\left(\theta_{h}^{n}-\theta_{A} c_{h}^{n}-\theta_{B}\left(1-c_{h}^{n}\right), x_{h}\right), \quad \forall x_{h} \in X_{h} .
\end{array}\right.
$$

Find $\theta_{h}^{n+1} \in X_{h}$ and $c_{h}^{n+1} \in X_{h}$ as solutions of the decoupled variational problems:

$$
\begin{gathered}
C_{V}\left(\frac{\theta_{h}^{n+1}-\theta_{h}^{n}}{k}, x_{h}\right)+\left(K_{1}^{h}\left(\phi_{h}^{n+1}\right) \nabla \theta_{h}^{n+1}, \nabla x_{h}\right)=-\frac{l}{2}\left(\frac{\phi_{h}^{n+1}-\phi_{h}^{n}}{k}, x_{h}\right), \quad \forall x_{h} \in X_{h}, \\
\left\{\begin{array}{l}
\left(\frac{c_{h}^{n+1}-c_{h}^{n}}{k}, x_{h}\right)+\left(K_{2}^{h}\left(\phi_{h}^{n+1}\right) \nabla c_{h}^{n+1}, \nabla x_{h}\right) \\
=-M\left(K_{2}^{h}\left(\phi_{h}^{n+1}\right)\left[P_{0} c_{h}^{n}\right]_{T}\left(1-\left[P_{0} c_{h}^{n}\right]_{T}\right) \nabla \phi_{h}^{n}, \nabla x_{h}\right), \quad \forall x_{h} \in X_{h} .
\end{array}\right.
\end{gathered}
$$


Here $K_{1}^{h}=K_{1}+g_{1}(h), K_{2}^{h}=K_{2}+g_{2}(h)$, where $g_{i}$ are positive functions to be chosen later, and $P_{0}$ is the $L^{2}$ orthogonal projector onto $X_{h}^{0}$, where $X_{h}^{0}$ is the finite element space of piecewise constant functions, and $[\cdot]_{T}$ is a truncation operator defined from $X_{h}^{0}$ into $X_{h}^{0}$ as follows: Given $x_{h} \in X_{h}^{0}$, then $\left[x_{h}\right]_{T} \in X_{h}^{0}$ such that

$$
\forall K \in \mathcal{T}_{h},\left.\quad\left[x_{h}\right]_{T}\right|_{K}=\left\{\begin{array}{lll}
\left.x_{h}\right|_{K} & \text { if } & \left.x_{h}\right|_{K} \in[0,1], \\
0 & \text { if } & \left.x_{h}\right|_{K}<0, \\
1 & \text { if } & \left.x_{h}\right|_{K}>1 .
\end{array}\right.
$$

Since (2.2) and (2.3) are quadratic linear systems, it is easy to check the existence and uniqueness of solutions. On the other hand, (2.1) is a discrete nonlinear variational problem and its existence and uniqueness can be proved as follows: We define

$$
J\left(\phi_{h}\right)=\frac{\alpha}{2 k} \int_{\Omega}\left|\phi_{h}\right|^{2}+\frac{1}{2} \int_{\Omega}\left(\left|\nabla \phi_{h}\right|^{2}+\left|\phi_{h}\right|^{2}\right)+\frac{1}{8 \varepsilon^{2}} \int_{\Omega}\left|\phi_{h}\right|^{4}-\int_{\Omega} g \phi_{h},
$$

where $g=\frac{\alpha}{k} \phi_{h}^{n}+\left(\frac{1}{2 \varepsilon^{2}}+1\right) \phi_{h}^{n}+\frac{\beta}{\varepsilon^{2}}\left(\theta_{h}^{n}-\theta_{A} c_{h}^{n}-\theta_{B}\left(1-c_{h}^{n}\right)\right)$. Clearly, $J$ is a strictly convex functional on $X_{h}$, then the minimum problem $\min _{\phi_{h} \in X_{h}} J\left(\phi_{h}\right)$ has a unique solution characterized by its Euler equation (2.1).

We will denote by $C$ generic positive constants always independent of the discretization parameters $h$ and $k$.

\section{A PRIORI ESTIMATES AND WEAK CONVERGENCES}

Let us add and subtract the term $\frac{1}{2 \varepsilon^{2}}\left(\phi_{h}^{n+1}, x_{h}\right)$ to the left-hand side of (2.1) in order to rewrite (2.1) with respect to the so-called Ginzburg-Landau function $f(\phi)=\frac{1}{2 \varepsilon^{2}}\left(\phi^{2}-1\right) \phi$ which has the potential function $F(\phi)=\frac{1}{8 \varepsilon^{2}}\left(\phi^{2}-1\right)^{2}$, that is, $f(\phi)=\nabla_{\phi} F(\phi)$. Then, (2.1) is rewritten as:

$$
\left\{\begin{array}{l}
\alpha\left(\frac{\phi_{h}^{n+1}-\phi_{h}^{n}}{k}, x_{h}\right)+\left(\nabla \phi_{h}^{n+1}, \nabla x_{h}\right)+\left(\phi_{h}^{n+1}, x_{h}\right) \\
+\left(f\left(\phi_{h}^{n+1}\right), x_{h}\right)+\frac{1}{2 \varepsilon^{2}}\left(\phi_{h}^{n+1}-\phi_{h}^{n}, x_{h}\right)=\left(\phi_{h}^{n}, x_{h}\right) \\
+\frac{\beta}{\varepsilon^{2}}\left(\theta_{h}^{n}-\theta_{A} c_{h}^{n}-\theta_{B}\left(1-c_{h}^{n}\right), x_{h}\right) \quad \forall x_{h} \in X_{h} .
\end{array}\right.
$$

It is easy to check that if we select $\phi_{h}^{0}=I_{h} \phi_{0}, \theta_{h}^{0}=I_{h} \theta_{0}$ and $c_{h}^{0}=I_{h} c_{0}$ as initial approximations, where $I_{h}$ is an interpolation operator into $X_{h}$ satisfying stability properties in the $L^{2}, L^{4}$ and $H^{1}$ norms, it follows that there exists a constant $C_{2}>0$ (independent of $\varepsilon$ ) such that

$$
\frac{2 C_{V} \beta}{l \varepsilon^{2}}\left|\theta_{h}^{0}\right|^{2}+\left|c_{h}^{0}\right|^{2}+1+\left\|\phi_{h}^{0}\right\|_{H^{1}(\Omega)}^{2}+\frac{1}{4 \varepsilon^{2}} \int_{\Omega}\left(\left|\phi_{h}^{0}\right|^{2}-1\right)^{2} \leq \frac{C_{2}}{\varepsilon^{2}} .
$$

For instance, this is true when $I_{h}$ is the $L^{2}$-projector onto $X_{h}$, or $I_{h}$ is the Clément or Scott-Zhang regularization operator.

Let us denote by $|\cdot|$ the $L^{2}(\Omega)$-norm and by $\|\cdot\|_{H^{1}(\Omega)}$ the $H^{1}(\Omega)$-norm. With such a notation we establish the following stability result. 
Lemma 3.1. Assume $\phi_{0} \in H^{1}(\Omega), \theta_{0} \in L^{2}(\Omega)$ and $c_{0} \in L^{2}(\Omega)$ such that $0 \leq c_{0} \leq 1$ a.e. in $\bar{\Omega}$. Then, for each $k$ such that $\frac{\beta k}{\varepsilon^{2}}$ is sufficiently small, the discrete solution of scheme (2.1)-(2.3) satisfies the following estimates:
(i) $\max _{0 \leq n \leq N}\left\|\phi_{h}^{n}\right\|_{H^{1}(\Omega)}^{2} \leq C$,
(ii) $\sum_{n=0}^{N-1}\left\|\phi_{h}^{n+1}-\phi_{h}^{n}\right\|_{H^{1}(\Omega)}^{2} \leq C$,
(iii) $\quad k \sum_{n=0}^{N-1}\left|\frac{\phi_{h}^{n+1}-\phi_{h}^{n}}{k}\right|^{2} \leq C$,
(iv) $\max _{0 \leq n \leq N}\left|\theta_{h}^{n}\right|^{2} \leq C$,
(v) $\sum_{n=0}^{N-1}\left|\theta_{h}^{n+1}-\theta_{h}^{n}\right|^{2} \leq C$,
(vi) $\quad k \sum_{n=0}^{N-1}\left|\sqrt{K_{1}^{h}\left(\phi_{h}^{n+1}\right)} \nabla \theta_{h}^{n+1}\right|^{2} \leq C$,
(vii) $\max _{0 \leq n \leq N}\left|c_{h}^{n}\right|^{2} \leq C$
(viii) $\quad \sum_{n=0}^{N-1}\left|c_{h}^{n+1}-c_{h}^{n}\right|^{2} \leq C$,
(ix) $k \sum_{n=0}^{N-1}\left|\sqrt{K_{2}^{h}\left(\phi_{h}^{n+1}\right)} \nabla c_{h}^{n+1}\right|^{2} \leq C$,

where $C>0$ depends on $\varepsilon$ and the data $\left(\phi_{0}, \theta_{0}, c_{0}\right)$ but is independent of $(h, k)$.

Proof. Let $x_{h}=\frac{4 \beta}{l \varepsilon^{2}} k \theta_{h}^{n+1}$ and $x_{h}=2 k c_{h}^{n+1}$ be test functions in (2.2) and (2.3), respectively. Now by using the identity $(a-b, 2 a)=|a|^{2}-|b|^{2}+|a-b|^{2}$ and bounding adequately the right-hand side, we have

$$
\begin{aligned}
& \frac{2 C_{V} \beta}{l \varepsilon^{2}}\left(\left|\theta_{h}^{n+1}\right|^{2}-\left|\theta_{h}^{n}\right|^{2}+\left|\theta_{h}^{n+1}-\theta_{h}^{n}\right|^{2}\right)+\frac{4 \beta}{l \varepsilon^{2}} k\left|\sqrt{K_{1}^{h}\left(\phi_{h}^{n+1}\right)} \nabla \theta_{h}^{n+1}\right|^{2} \\
& =-\frac{2 \beta}{\varepsilon^{2}} k\left(\frac{\phi_{h}^{n+1}-\phi_{h}^{n}}{k}, \theta_{h}^{n}+\left(\theta_{h}^{n+1}-\theta_{h}^{n}\right)\right) \\
& \leq-\frac{2 \beta}{\varepsilon^{2}} k\left(\frac{\phi_{h}^{n+1}-\phi_{h}^{n}}{k}, \theta_{h}^{n}\right)+\frac{\alpha}{2} k\left|\frac{\phi_{h}^{n+1}-\phi_{h}^{n}}{k}\right|^{2}+\frac{2 \beta^{2}}{\alpha \varepsilon^{4}} k\left|\theta_{h}^{n+1}-\theta_{h}^{n}\right|^{2}, \\
& \left|c_{h}^{n+1}\right|^{2}-\left|c_{h}^{n}\right|^{2}+\left|c_{h}^{n+1}-c_{h}^{n}\right|^{2}+k\left|\sqrt{K_{2}^{h}\left(\phi_{h}^{n+1}\right)} \nabla c_{h}^{n+1}\right|^{2} \leq C k\left|\nabla \phi_{h}^{n}\right|^{2} .
\end{aligned}
$$

By choosing $\frac{\beta k}{\varepsilon^{2}}$ sufficiently small to control the last term on the right-hand side of (3.3), this inequality reduces to

$$
\begin{aligned}
\frac{2 C_{V} \beta}{l \varepsilon^{2}}\left(\left|\theta_{h}^{n+1}\right|^{2}-\left|\theta_{h}^{n}\right|^{2}+\frac{1}{2}\left|\theta_{h}^{n+1}-\theta_{h}^{n}\right|^{2}\right)+\frac{4 \beta}{l \varepsilon^{2}} k\left|\sqrt{K_{1}^{h}\left(\phi_{h}^{n+1}\right)} \nabla \theta_{h}^{n+1}\right|^{2} \leq \\
\quad-\frac{2 \beta}{\varepsilon^{2}} k\left(\frac{\phi_{h}^{n+1}-\phi_{h}^{n}}{k}, \theta_{h}^{n}\right)+\frac{\alpha}{2} k\left|\frac{\phi_{h}^{n+1}-\phi_{h}^{n}}{k}\right|^{2} .
\end{aligned}
$$

Next, take $x_{h}=2 k \frac{\phi_{h}^{n+1}-\phi_{h}^{n}}{k}$ as a test function in (2.1), it follows that

$$
\begin{array}{r}
\alpha k\left|\frac{\phi_{h}^{n+1}-\phi_{h}^{n}}{k}\right|^{2}+\left(\left\|\phi_{h}^{n+1}\right\|_{H^{1}(\Omega)}^{2}-\left\|\phi_{h}^{n}\right\|_{H^{1}(\Omega)}^{2}+\left\|\phi_{h}^{n+1}-\phi_{h}^{n}\right\|_{H^{1}(\Omega)}^{2}\right)+2\left(f\left(\phi_{h}^{n+1}\right), \phi_{h}^{n+1}-\phi_{h}^{n}\right) \\
+\frac{1}{\varepsilon^{2}}\left|\phi_{h}^{n+1}-\phi_{h}^{n}\right|^{2} \leq \frac{2 \beta}{\varepsilon^{2}} k\left(\theta_{h}^{n}, \frac{\phi_{h}^{n+1}-\phi_{h}^{n}}{k}\right)+C k\left|\phi_{h}^{n}\right|^{2}+C \frac{\beta^{2}}{\alpha \varepsilon^{4}} k\left(\left|c_{h}^{n}\right|^{2}+1\right),
\end{array}
$$

where $C$ is a constant independent of $\varepsilon$. 
Now, using again the identity $(a-b, a)=\frac{1}{2}\left(|a|^{2}-|b|^{2}+|a-b|^{2}\right)$ twice, we can rewrite

$$
\begin{aligned}
2\left(f\left(\phi_{h}^{n+1}\right), \phi_{h}^{n+1}-\phi_{h}^{n}\right)= & \frac{1}{2 \varepsilon^{2}} \int_{\Omega}\left(\left(\phi_{h}^{n+1}\right)^{2}-1\right)\left(\left(\phi_{h}^{n+1}\right)^{2}-\left(\phi_{h}^{n}\right)^{2}+\left(\phi_{h}^{n+1}-\phi_{h}^{n}\right)^{2}\right) \\
= & 2 \int_{\Omega}\left(F\left(\phi_{h}^{n+1}\right)-F\left(\phi_{h}^{n}\right)+\frac{1}{8 \varepsilon^{2}}\left(\left(\phi_{h}^{n+1}\right)^{2}-\left(\phi_{h}^{n}\right)^{2}\right)^{2}\right) \\
& +\frac{1}{2 \varepsilon^{2}} \int_{\Omega}\left(\left(\phi_{h}^{n+1}\right)^{2}-1\right)\left|\phi_{h}^{n+1}-\phi_{h}^{n}\right|^{2} .
\end{aligned}
$$

Note that the negative term on the right-hand side of (3.7) can be absorbed by the last term on the left-hand side of (3.6). This property can be summarized as

$$
\frac{1}{2 \varepsilon^{2}}\left(\left(\phi_{h}^{n+1}\right)^{3}-\phi_{h}^{n}\right)\left(\phi_{h}^{n+1}-\phi_{h}^{n}\right) \geq F\left(\phi_{h}^{n+1}\right)-F\left(\phi_{h}^{n}\right)
$$

which is an appropriate discrete version of the equality $f(\phi) \phi_{t}=F(\phi)_{t}$.

Next, if we add up (3.4), (3.5) and (3.6), the first term on the right hand side of (3.5) and (3.6) disappears, and we get

$$
\begin{aligned}
& \frac{2 C_{V} \beta}{l \varepsilon^{2}}\left(\left|\theta_{h}^{n+1}\right|^{2}-\left|\theta_{h}^{n}\right|^{2}+\frac{1}{2}\left|\theta_{h}^{n+1}-\theta_{h}^{n}\right|^{2}\right)+\frac{4 \beta}{l \varepsilon^{2}} k\left|\sqrt{K_{1}^{h}\left(\phi_{h}^{n+1}\right)} \nabla \theta_{h}^{n+1}\right|^{2} \\
& +\left(\left|c_{h}^{n+1}\right|^{2}+1\right)-\left(\left|c_{h}^{n}\right|^{2}+1\right)+\left|c_{h}^{n+1}-c_{h}^{n}\right|^{2}+k\left|\sqrt{K_{2}^{h}\left(\phi_{h}^{n+1}\right)} \nabla c_{h}^{n+1}\right|^{2} \\
& +\frac{\alpha}{2} k\left|\frac{\phi_{h}^{n+1}-\phi_{h}^{n}}{k}\right|^{2}+\left(\left\|\phi_{h}^{n+1}\right\|_{H^{1}(\Omega)}^{2}-\left\|\phi_{h}^{n}\right\|_{H^{1}(\Omega)}^{2}+\left\|\phi_{h}^{n+1}-\phi_{h}^{n}\right\|_{H^{1}(\Omega)}^{2}\right) \\
& +2 \int_{\Omega}\left(F\left(\phi_{h}^{n+1}\right)-F\left(\phi_{h}^{n}\right)\right) \leq C k\left\|\phi_{h}^{n}\right\|_{H^{1}(\Omega)}^{2}+C \frac{\beta^{2}}{\alpha \varepsilon^{4}} k\left(\left|c_{h}^{n}\right|^{2}+1\right) .
\end{aligned}
$$

Finally, by summing over $n$ the discrete Gronwall lemma and the initial bound (3.2) provide the desired estimates, and this completes the proof.

Remark 3.2. Since the initial estimates have order $O\left(\varepsilon^{-2}\right)$, see (3.2), then the stability estimates obtained in Lemma 3.1 are of order $O\left(\varepsilon^{-2} \mathrm{e}^{C \beta^{2} \varepsilon^{-4}}\right)$ for the variables $\left(\phi, \frac{\sqrt{\beta}}{\varepsilon} \theta, c\right)$. As $\beta$ is of order $O(\varepsilon)$, the order reduces to $O\left(\varepsilon^{-2} \mathrm{e}^{C \varepsilon^{-2}}\right)$. In particular, these estimates would be independent of $\varepsilon$ if $\beta$ were of order $O\left(\varepsilon^{2}\right)$ and considering an initial bound (3.2) independent of $\varepsilon$. Furthermore, if we truncate the discrete concentration $c_{h}^{n}$ in (2.1) as made in (2.3), that is replacing $\frac{\beta}{\varepsilon^{2}}\left(\theta_{h}^{n}-\theta_{A} c_{h}^{n}-\theta_{B}\left(1-c_{h}^{n}\right), x_{h}\right)$ by $\frac{\beta}{\varepsilon^{2}}\left(\theta_{h}^{n}-\theta_{A}\left[P_{0} c_{h}^{n}\right]_{T}-\theta_{B}\left(1-\left[P_{0} c_{h}^{n}\right]_{T}\right), x_{h}\right)$, this modified scheme has stability estimates of order $O\left(\varepsilon^{-2}+\beta^{2} \varepsilon^{-4}\right)$.

Remark 3.3. Observe that in this nonlinear scheme, we have used a first-order semi-implicit approximation of the Ginzburg-Landau function $f(\phi)$, which provides a stationary problem to solve in each time step, identified with the critical point of a convex functional (see (2.4)). Moreover, this approximation verifies the property (3.8). For instance, if we use the first-order implicit approximation $f\left(\phi_{h}^{n+1}\right)$, then the associated stationary problem (of Allen-Cahn type) is related to the critical points of a non-convex functional and the property (3.8) in not verified, because a negative term appears on the right-hand side. To be more concrete, it follows that

$$
f\left(\phi_{h}^{n+1}\right)\left(\phi_{h}^{n+1}-\phi_{h}^{n}\right) \geq F\left(\phi_{h}^{n+1}\right)-F\left(\phi_{h}^{n}\right)-\frac{1}{4 \varepsilon^{2}}\left(\phi_{h}^{n+1}-\phi_{h}^{n}\right)^{2} .
$$


Consider the linear operator $L_{h}: X_{h} \rightarrow X_{h}$ defined as:

$$
\left(L_{h} \phi_{h}, x_{h}\right)=\left(\nabla \phi_{h}, \nabla x_{h}\right)+\left(\phi_{h}, x_{h}\right) \quad \forall x_{h} \in X_{h}
$$

Then, the discrete phase-field equation (2.1) can be rewritten as:

$$
\left\{\begin{array}{l}
\left(\frac{\phi_{h}^{n+1}-\phi_{h}^{n}}{k}, x_{h}\right)+\frac{1}{\alpha}\left(L_{h} \phi_{h}^{n+1}, x_{h}\right)+\frac{1}{2 \alpha \varepsilon^{2}}\left(\left(\phi_{h}^{n+1}\right)^{3}, x_{h}\right) \\
=\left(\frac{1}{2 \alpha \varepsilon^{2}}+\frac{1}{\alpha}\right)\left(\phi_{h}^{n}, x_{h}\right)+\frac{\beta}{\alpha \varepsilon^{2}}\left(\theta_{h}^{n}-\theta_{A} c_{h}^{n}-\theta_{B}\left(1-c_{h}^{n}\right), x_{h}\right), \quad \forall x_{h} \in X_{h} .
\end{array}\right.
$$

Taking $x_{h}=L_{h} \phi_{h}^{n+1}$ as a test function in (3.10) and using the estimates of Lemma 3.1, the following result can be established.

Corollary 3.4. Under the hypotheses of Lemma 3.1, it holds

$$
k \sum_{n=0}^{N-1}\left|L_{h} \phi_{h}^{n+1}\right|^{2} \leq C
$$

On the other hand, since $K_{1}(\cdot) \leq b_{1}$ and $K_{2}(\cdot) \leq b_{2}$, from $(\boldsymbol{v} \boldsymbol{i})$ and $(\boldsymbol{i x})$ of Lemma 3.1 we also have

$$
k \sum_{n=0}^{N-1}\left|K_{1}^{h}\left(\phi_{h}^{n+1}\right) \nabla \theta_{h}^{n+1}\right|^{2} \leq C, \quad k \sum_{n=0}^{N-1}\left|K_{2}^{h}\left(\phi_{h}^{n+1}\right) \nabla c_{h}^{n+1}\right|^{2} \leq C .
$$

Definition 3.5. We define $\phi_{h, k}$ (respectively $\widehat{\phi}_{h, k}$ ) as the piecewise constant functions in time taking values $\phi_{h}^{n+1}$ on $\left(t_{n}, t_{n+1}\right]$ (respectively $\left.\phi_{h}^{n}\right)$. Analogously, we define $\theta_{h, k}, \widehat{\theta}_{h, k}$, and $c_{h, k}, \widehat{c}_{h, k}$. Moreover, we define $\widetilde{\phi}_{h, k}$, $\widetilde{\theta}_{h, k}, \widetilde{c}_{h, k} \in C^{0}\left([0, T] ; X_{h}\right)$ as the piecewise linear functions in time such that $\widetilde{\phi}_{h, k}\left(t_{n}\right)=\phi_{h}^{n}, \widetilde{\theta}_{h, k}\left(t_{n}\right)=\theta_{h}^{n}$, $\widetilde{c}_{h, k}\left(t_{n}\right)=c_{h}^{n}$, respectively.

An easy consequence of the previous definition, Lemma 3.1 and Corollary 3.4 is the following result.

Lemma 3.6. Under the hypotheses of Lemma 3.1, the following estimates hold:

$$
\begin{array}{rll}
\left\{\theta_{h, k}\right\}_{h, k},\left\{\widehat{\theta}_{h, k}\right\}_{h, k},\left\{\widetilde{\theta}_{h, k}\right\}_{h, k} & \text { is bounded in } & L^{\infty}\left(0, T ; L^{2}(\Omega)\right), \\
\left\{c_{h, k}\right\}_{h, k},\left\{\widehat{c}_{h, k}\right\}_{h, k},\left\{\widetilde{c}_{h, k}\right\}_{h, k} & \text { is bounded in } & L^{\infty}\left(0, T ; L^{2}(\Omega)\right), \\
\left\{\phi_{h, k}\right\}_{h, k},\left\{\widehat{\phi}_{h, k}\right\}_{h, k},\left\{\widetilde{\phi}_{h, k}\right\}_{h, k} & \text { is bounded in } & L^{\infty}\left(0, T ; H^{1}(\Omega)\right), \\
\left\{K_{1}^{h}\left(\phi_{h, k}\right) \nabla \theta_{h, k}\right\}_{h, k} & \text { is bounded in } & L^{2}\left(0, T ; L^{2}(\Omega)\right), \\
\left\{K_{2}^{h}\left(\phi_{h, k}\right) \nabla c_{h, k}\right\}_{h, k} & \text { is bounded in } & L^{2}\left(0, T ; L^{2}(\Omega)\right), \\
\left\{\frac{\mathrm{d}}{\mathrm{d} t} \widetilde{\phi}_{h, k}\right\}_{h, k} & \text { is bounded in } & L^{2}\left(0, T ; L^{2}(\Omega)\right) . \\
\left\{L_{h} \phi_{h, k}\right\}_{h, k} & \text { is bounded in } & L^{2}\left(0, T ; L^{2}(\Omega)\right) .
\end{array}
$$


In addition, there exist a subsequence of $(h, k)$ (denoted in the same way) and limit functions $\phi, \theta, c, w, J_{1}$ and $J_{2}$ verifying the following weak convergences as $(h, k) \rightarrow 0$ :

$$
\begin{gathered}
\theta_{h, k} \rightarrow \theta, \quad \widehat{\theta}_{h, k} \rightarrow \theta, \quad \widetilde{\theta}_{h, k} \rightarrow \theta \quad \text { in } L^{\infty}\left(0, T ; L^{2}(\Omega)\right) \text {-weak* } \\
c_{h, k} \rightarrow c, \quad \widehat{c}_{h, k} \rightarrow c, \quad \widetilde{c}_{h, k} \rightarrow c \quad \text { in } L^{\infty}\left(0, T ; L^{2}(\Omega)\right) \text {-weak }{ }^{*}, \\
\phi_{h, k} \rightarrow \phi, \quad \widehat{\phi}_{h, k} \rightarrow \phi, \quad \widetilde{\phi}_{h, k} \rightarrow \phi \quad \text { in } L^{\infty}\left(0, T ; H^{1}(\Omega)\right) \text {-weak } \\
\frac{\mathrm{d}}{\mathrm{d} t} \widetilde{\phi}_{h, k} \rightarrow \frac{\mathrm{d}}{\mathrm{d} t} \phi \quad \text { in } L^{2}\left(0, T ; L^{2}(\Omega)\right) \text {-weak }, \\
L_{h} \phi_{h, k} \rightarrow w \quad \text { in } L^{2}\left(0, T ; L^{2}(\Omega)\right) \text {-weak, } \\
K_{1}^{h}\left(\phi_{h, k}\right) \nabla \theta_{h, k} \rightarrow J_{1} \quad \text { in } L^{2}\left(0, T ; L^{2}(\Omega)\right) \text {-weak }, \\
K_{2}^{h}\left(\phi_{h, k}\right) \nabla c_{h, k} \rightarrow J_{2} \quad \text { in } L^{2}\left(0, T ; L^{2}(\Omega)\right) \text {-weak. }
\end{gathered}
$$

So far, neither the $H^{2}(\Omega)$-regularity for the Neumann problem (3.19) nor the quasi-uniform property of the triangulation $\mathcal{T}_{h}$ of $\Omega$ has not been necessary to impose. Now, imposing these hypotheses, the next corollary provides a "discrete interpolation" inequality which plays an important role in getting a compactness result (see the proof of Prop. 4.3) that we will use to pass to the limit in (2.3) (respectively, in (6.3) for the linear scheme).

Corollary 3.7. Under the hypotheses of Theorem 1.3, it follows that

$$
\left\|\phi_{h}^{n+1}\right\|_{W^{1,3}(\Omega)} \leq C\left|\phi_{h}^{n+1}\right|^{1 / 2}\left|L_{h} \phi_{h}^{n+1}\right|^{1 / 2}
$$

where $C>0$ is independent of $h$ and $k$.

Proof. Let $\phi(h) \in H^{2}(\Omega)$ be the solution to the problem

$$
-\Delta \phi(h)+\phi(h)=L_{h} \phi_{h}^{n+1} \text { in } \Omega, \quad \frac{\partial \phi(h)}{\partial \boldsymbol{n}}=0 \text { on } \Gamma .
$$

We now suppose that problem (3.19) has the regularity property $\|\phi(h)\|_{H^{2}(\Omega)} \leq C\left|L_{h} \phi_{h}^{n+1}\right|$ (such a condition holds if, for instance, $\Omega$ is a convex polygon). From (3.9) and (3.19), we have

$$
\left(\nabla \phi(h)-\nabla \phi_{h}^{n+1}, \nabla x_{h}\right)+\left(\phi(h)-\phi_{h}^{n+1}, x_{h}\right)=0 \quad \forall x_{h} \in X_{h}
$$

hence $\phi_{h}^{n+1}$ can be interpreted as the $H^{1}$-projection of $\phi(h)$ onto $X_{h}$. Then, the estimate $\left\|\phi_{h}^{n+1}\right\|_{W^{1,3}(\Omega)} \leq$ $C\|\phi(h)\|_{W^{1,3}(\Omega)}$ holds for a constant $C>0$ independent of $h$ (see [3], Chap. 8, and [11]). Thus,

$$
\left\|\phi_{h}^{n+1}\right\|_{W^{1,3}(\Omega)}^{2} \leq C\|\phi(h)\|_{H^{1}(\Omega)}\|\phi(h)\|_{H^{2}(\Omega)} \leq C\|\phi(h)\|_{H^{1}(\Omega)}\left|L_{h} \phi_{h}^{n+1}\right| .
$$

Therefore, it remains to bound $\|\phi(h)\|_{H^{1}(\Omega)} \leq C\left\|\phi_{h}^{n+1}\right\|_{H^{1}(\Omega)}$ in order to obtain (3.18). Indeed, we write

$$
\|\phi(h)\|_{H^{1}(\Omega)} \leq\left\|\phi(h)-\phi_{h}^{n+1}\right\|_{H^{1}(\Omega)}+\left\|\phi_{h}^{n+1}\right\|_{H^{1}(\Omega)} \leq C h\left|L_{h} \phi_{h}^{n+1}\right|+\left\|\phi_{h}^{n+1}\right\|_{H^{1}(\Omega)},
$$

where in the last bound we have used that $\left\|\phi(h)-\phi_{h}^{n+1}\right\|_{H^{1}(\Omega)} \leq C h\|\phi(h)\|_{H^{2}(\Omega)}$. Finally, the estimate $\left|L_{h} \phi_{h}^{n+1}\right| \leq C \frac{1}{h}\left\|\phi_{h}^{n+1}\right\|_{H^{1}(\Omega)}$ can be deduced by taking $\phi_{h}=\phi_{h}^{n+1}$ and $x_{h}=L_{h} \phi_{h}^{n+1}$ in (3.9) and using the inverse inequality $\left\|L_{h} \phi_{h}^{n+1}\right\|_{H^{1}(\Omega)} \leq C \frac{1}{h}\left|L_{h} \phi_{h}^{n+1}\right|$ (here the quasi-uniform property of the triangulation is used).

A straightforward application of (3.18) shows that

$$
\left\{\phi_{h, k}\right\}_{h, k} \text { is bounded in } L^{4}\left(0, T ; W^{1,3}(\Omega)\right) \text {. }
$$




\section{Strong CONVERGENCES}

Let us show some compactness results in order to identify firstly $w=-\Delta \phi+\phi, J_{1}=\nabla\left(K_{1}(\phi) \theta\right)-\theta \nabla K_{1}(\phi)$ and $J_{2}=\nabla\left(K_{2}(\phi) \theta\right)-\theta \nabla K_{2}(\phi)$ and then to pass to the limit as $(h, k) \rightarrow 0$.

\subsection{Compactness for phase-field sequences}

First of all, since $\left\{\widetilde{\phi}_{h, k}\right\}_{h, k}$ is bounded in $L^{\infty}\left(0, T, H^{1}(\Omega)\right)$ and $\left\{\frac{\mathrm{d}}{\mathrm{d} t} \widetilde{\phi}_{h, k}\right\}_{h, k}$ is bounded in $L^{2}\left(0, T ; L^{2}(\Omega)\right)$, a compactness theorem of Aubin-Lions type [13] provides

$$
\widetilde{\phi}_{h, k} \rightarrow \phi \quad \text { in } C\left(0, T ; L^{p}(\Omega)\right) \text { strongly as }(h, k) \rightarrow 0,
$$

with $p<6$. Moreover, owing to Lemma 3.1

$$
\left\|\widetilde{\phi}_{h, k}-\phi_{h, k}\right\|_{L^{2}\left(0, T ; L^{2}(\Omega)\right)}^{2} \leq\left\|\widehat{\phi}_{h, k}-\phi_{h, k}\right\|_{L^{2}\left(0, T ; L^{2}(\Omega)\right)}^{2}=k \sum_{n=0}^{N-1}\left|\phi_{h}^{n+1}-\phi_{h}^{n}\right|^{2} \leq C k .
$$

Therefore, $\phi_{h, k} \rightarrow \phi, \widehat{\phi}_{h, k} \rightarrow \phi$ in $L^{2}\left(0, T ; L^{2}(\Omega)\right)$ strongly as $(h, k) \rightarrow 0$. As $\{\phi\}_{h, k}$ and $\{\widehat{\phi}\}_{h, k}$ are bounded in $L^{\infty}\left(0, T ; H^{1}(\Omega)\right)$, Sobolev's imbedding gives us the strong convergences

$$
\phi_{h, k}, \widehat{\phi}_{h, k} \rightarrow \phi \quad \text { in } L^{q}\left(0, T ; L^{p}(\Omega)\right) \text { strongly as }(h, k) \rightarrow 0,
$$

with $q<\infty$ and $p<6$.

To prove the compactness of $\left\{\phi_{h, k}\right\}_{h, k}$ in $L^{2}\left(0, T ; H^{1}(\Omega)\right)$ we firstly must identify $w=-\Delta \phi+\phi$. Indeed, we consider $\eta \in C_{c}^{\infty}(Q)$ and choose $\eta_{h}^{n} \in X_{h}$ a suitable approximation of $\eta\left(t_{n}\right)$ such that $\eta_{h, k} \rightarrow \eta$ in $L^{2}\left(0, T ; H^{1}(\Omega)\right)$ strongly as $(h, k) \rightarrow 0$ (here $\eta_{h, k}$ is defined by $\eta_{h}^{n}$ as in Def. 3.5). Then, setting $\phi_{h}=\phi_{h}^{n+1}$ and $x_{h}=\eta_{h}^{n+1}$ in the definition of $L_{h}(3.9)$, multiplying by $k$ and summing over $n$ and tending $(h, k) \rightarrow 0$, one sees that

$$
\int_{Q}(\nabla \phi, \nabla \eta)+(\phi, \eta) \leftarrow \int_{Q}\left(\nabla \phi_{h, k}, \nabla \eta_{h, k}\right)+\left(\phi_{h, k}, \eta_{h, k}\right)=\int_{Q}\left(L_{h} \phi_{h, k}, \eta_{h, k}\right) \rightarrow \int_{Q}(w, \eta) .
$$

Therefore, it is clear that $w=-\Delta \phi+\phi$ in $L^{2}(\Omega)$. Next, taking $\eta \in C^{\infty}(Q)$ and proceeding in the same manner, we recover the boundary condition $\frac{\partial \phi}{\partial \boldsymbol{n}}=0$ on $\Sigma$.

Now, we continue to get the compactness of $\left\{\phi_{h, k}\right\}_{h, k}$ in $L^{2}\left(0, T ; H^{1}(\Omega)\right)$. Considering $\phi_{h}=\phi_{h}^{n+1}$ and $x_{h}=\phi_{h}^{n+1}$ in (3.9), multiplying by $k$ and summing over $n$, this results

$$
\int_{0}^{T}\left\|\phi_{h, k}\right\|_{H^{1}(\Omega)}^{2}=\int_{0}^{T}\left(L_{h} \phi_{h, k}, \phi_{h, k}\right) \longrightarrow \int_{0}^{T}(-\Delta \phi+\phi, \phi)=\int_{0}^{T}\|\phi\|_{H^{1}(\Omega)}^{2} \quad \text { as }(h, k) \rightarrow 0,
$$

because of $\phi_{h, k} \rightarrow \phi$ strongly in $L^{2}\left(0, T ; L^{2}(\Omega)\right)$ and $\left\{L_{h} \phi_{h, k}\right\}_{h, k} \rightarrow-\Delta \phi+\phi$ weakly in $L^{2}\left(0, T ; L^{2}(\Omega)\right.$.

Therefore, one can obtain the convergence $\left\|\phi_{h, k}\right\|_{L^{2}\left(0, T ; H^{1}(\Omega)\right)} \rightarrow\|\phi\|_{L^{2}\left(0, T ; H^{1}(\Omega)\right)}$ as $(h, k) \rightarrow 0$. Consequently, since $\phi_{h, k} \rightarrow \phi$ weakly* in $L^{2}\left(0, T ; H^{1}(\Omega)\right)$, one has

$$
\left\|\phi_{h, k}-\phi\right\|_{L^{2}\left(0, T ; H^{1}(\Omega)\right)} \rightarrow 0 \text { as }(h, k) \rightarrow 0 .
$$




\subsection{Compactness for temperature and concentration sequences}

Lemma 4.1. The following estimates hold

$$
\begin{aligned}
& \int_{0}^{T}\left\|\frac{\mathrm{d}}{\mathrm{d} t} \widetilde{\theta}_{h, k}(t)\right\|_{H^{1}(\Omega)^{\prime}}^{2} \mathrm{~d} t \leq C, \\
& \int_{0}^{T}\left\|\frac{\mathrm{d}}{\mathrm{d} t} \widetilde{c}_{h, k}(t)\right\|_{H^{1}(\Omega)^{\prime}}^{2} \mathrm{~d} t \leq C,
\end{aligned}
$$

where $C>0$ is independent of $(h, k)$.

Proof. Let $P_{h}$ be the orthogonal projector from $L^{2}(\Omega)$ onto $X_{h}$. Let $x \in H^{1}(\Omega)$. Then, by taking $x_{h}=P_{h} x$ as a test function in (2.2), we obtain

$$
C_{V}\left(\frac{\theta_{h}^{n+1}-\theta_{h}^{n}}{k}, x\right) \leq C\left|K_{1}^{h}\left(\phi_{h}^{n+1}\right) \nabla \theta_{h}^{n+1}\right|\|x\|_{H^{1}(\Omega)}+\frac{l}{2}\left|\frac{\phi_{h}^{n+1}-\phi_{h}^{n}}{k}\right||x|,
$$

where we have used the following stability properties of the $L^{2}$-projector, $\left|P_{h} x\right| \leq|x|$ and $\left\|P_{h} x\right\|_{H^{1}} \leq C\|x\|_{H^{1}}$ (the stability in the $H^{1}$-norm can be obtained by means of a duality argument and comparing with the $H^{1}$-projector). Taking into account that $\frac{\mathrm{d}}{\mathrm{d} t} \widetilde{\theta}_{h, k}(t)=\frac{\theta_{h}^{n+1}-\theta_{h}^{n}}{k}$ for each $t \in\left(t_{n}, t_{n+1}\right)$, multiplying (4.3) by $k$, and adding up over $n$, estimate (4.1) is proved.

In a analogous way, one can be proved estimate (4.2).

As a consequence of Lemmas 3.6 and 4.1, one can use a compactness result [13] obtaining the following strong convergences as $(h, k) \rightarrow 0$ :

$$
\begin{gathered}
\widetilde{\theta}_{h, k} \rightarrow \theta \quad \text { strongly in } L^{2}\left(0, T ; H^{1}(\Omega)^{\prime}\right), \\
\widetilde{c}_{h, k} \rightarrow c \text { strongly in } L^{2}\left(0, T ; H^{1}(\Omega)^{\prime}\right) .
\end{gathered}
$$

In fact, due to Lemma 3.1, we also have that $\widehat{\theta}_{h, k}, \theta_{h, k} \rightarrow \theta$ strongly in $L^{2}\left(0, T ; H^{1}(\Omega)^{\prime}\right.$ ) (analogously $\widehat{c}_{h, k}, \widetilde{c}_{h, k} \rightarrow c$ strongly in $L^{2}\left(0, T ; H^{1}(\Omega)^{\prime}\right)$ as $\left.(h, k) \rightarrow 0\right)$, since

$$
\begin{aligned}
\left\|\widetilde{\theta}_{h, k}-\theta_{h, k}\right\|_{L^{2}\left(0, T ; H^{1}(\Omega)^{\prime}\right)}^{2} & \leq\left\|\theta_{h, k}-\widehat{\theta}_{h, k}\right\|_{L^{2}\left(0, T ; H^{1}(\Omega)^{\prime}\right)}^{2} \\
& \leq C\left\|\widehat{\theta}_{h, k}-\theta_{k, h}\right\|_{L^{2}\left(0, T ; L^{2}(\Omega)\right)}^{2}=C k \sum_{n=0}^{N-1}\left|\theta_{h}^{n+1}-\theta_{h}^{n}\right|^{2} \leq C k .
\end{aligned}
$$

To be able to pass to the limit in the nonlinear term of (2.3) we have to prove that the sequence $\left\{K_{2}^{h}\left(\phi_{h, k}\right)\left[P_{0} c_{h, k}\right]_{T}\left(1-\left[P_{0} c_{h, k}\right]_{T}\right)\right\}_{h, k}$ is weakly convergent to a certain limit which has to be identified later on.

First of all, we prove that $P_{0} c_{h, k}-c_{h, k}$ tends to zero as $(h, k)$ tend to zero under a certain condition for the auxiliary function $g_{2}(h)$ which defines $K_{2}^{h}$.

Proposition 4.2. If the function $g_{2}(h)$ given in Section 2 satisfies the condition $h / \sqrt{g_{2}(h)} \rightarrow 0$ as $h \rightarrow 0$, then the following convergence holds

$$
\left\|P_{0} \widehat{c}_{h, k}-\widehat{c}_{h, k}\right\|_{L^{2}\left(0, T ; L^{2}(\Omega)\right)} \rightarrow 0 \quad \text { as }(h, k) \rightarrow 0 .
$$

Proof. By using the fact that $K_{2}^{h}(\cdot) \geq g_{2}(h)$ and estimate $(\boldsymbol{i x})$ of Lemma 3.1, we get

$$
k \sum_{n=0}^{N-1}\left|\nabla c_{h}^{n+1}\right|^{2} \leq \frac{C}{g_{2}(h)} .
$$


By combining (4.6) and the error interpolation $\left|P_{0} c_{h}^{n}-c_{h}^{n}\right| \leq C h\left|\nabla c_{h}^{n}\right|$ applied for each $n \geq 1$, we see that

$$
\begin{aligned}
\left\|P_{0} \widehat{c}_{h, k}-\widehat{c}_{h, k}\right\|_{L^{2}\left(0, T ; L^{2}(\Omega)\right)}^{2} & \leq k\left|P_{0} c_{h}^{0}-c_{h}^{0}\right|^{2}+C h^{2}\left\|\nabla c_{h, k}\right\|_{L^{2}\left(0, T ; L^{2}(\Omega)\right)}^{2} \\
& \leq k\left|P_{0} c_{h}^{0}-c_{h}^{0}\right|^{2}+C \frac{h^{2}}{g_{2}(h)}
\end{aligned}
$$

hence $\left\|P_{0} \widehat{c}_{h, k}-\widehat{c}_{h, k}\right\|_{L^{2}\left(0, T ; L^{2}(\Omega)\right)}^{2} \rightarrow 0$ as $(h, k) \rightarrow 0$.

Finally, the following compactness result is established for the coefficients $K_{2}^{h}\left(\phi_{h, k}\right)\left[P_{0} \widehat{c}_{h, k}\right]_{T}\left(1-\left[P_{0} \widehat{c}_{h, k}\right]_{T}\right)$ which will achieve by using the fact that $\left[P_{0} \widehat{c}_{h, k}(\boldsymbol{x}, t)\right]_{T}=T_{0}^{1}\left(P_{0} \widehat{c}_{h, k}(\boldsymbol{x}, t)\right)$ a.e. $(\boldsymbol{x}, t) \in Q$ owing to $P_{0} c_{h, k}$ is a piecewise constant function, where $T_{0}^{1}$ is the pointwise truncation operator defined as

$$
T_{0}^{1} c(\boldsymbol{x}, t)= \begin{cases}c(\boldsymbol{x}, t) & \text { if } \quad c(\boldsymbol{x}, t) \in[0,1] \\ 0 & \text { if } \quad c(\boldsymbol{x}, t)<0 \\ 1 & \text { if } \quad c(\boldsymbol{x}, t)>1\end{cases}
$$

This compactness is not clear if we truncate $c_{h, k}$ by nodes, as was made in [8] for a nondegenerate mass diffusion Navier-Stokes model.

Proposition 4.3. The following convergence as $(h, k) \rightarrow 0$ holds, for each $p<\infty$ :

$$
K_{2}^{h}\left(\phi_{h, k}\right)\left[P_{0} \widehat{c}_{h, k}\right]_{T}\left(1-\left[P_{0} \widehat{c}_{h, k}\right]_{T}\right) \rightarrow K_{2}(\phi) T_{0}^{1} c\left(1-T_{0}^{1} c\right) \quad \text { in } L^{p}(Q) \text {-strong. }
$$

Proof. First of all, we prove that $K_{2}^{h}\left(\phi_{h, k}\right) c_{h, k} \rightarrow K_{2}(\phi) c$ strongly in $L^{2}\left(0, T ; L^{3 / 2}(\Omega)\right)$ as $(h, k) \rightarrow 0$. To this end, we define $\widetilde{\varphi}_{h, k}$ as the piecewise linear, globally continuous in time function taking the value $K_{2}^{h}\left(\phi_{h}^{n+1}\right) c_{h}^{n+1}$ at the time $t=t_{n+1}$. Our task now is to obtain an estimate in the $\left(W^{1, s}\right)^{\prime}$-norm for the time derivative of $\widetilde{\varphi}_{h, k}$, with $s>3$. Indeed, for $t \in\left(t_{n}, t_{n+1}\right)$,

$$
\frac{\mathrm{d}}{\mathrm{d} t} \widetilde{\varphi}_{h, k}(t)=\frac{K_{2}^{h}\left(\phi_{h}^{n+1}\right) c_{h}^{n+1}-K_{2}^{h}\left(\phi_{h}^{n}\right) c_{h}^{n}}{k}=K_{2}^{h}\left(\phi_{h}^{n+1}\right) \frac{c_{h}^{n+1}-c_{h}^{n}}{k}+c_{h}^{n} \frac{K_{2}^{h}\left(\phi_{h}^{n+1}\right)-K_{2}^{h}\left(\phi_{h}^{n}\right)}{k} .
$$

By the mean value theorem, the last term can be written as

$$
c_{h}^{n} \frac{K_{2}^{h}\left(\phi_{h}^{n+1}\right)-K_{2}^{h}\left(\phi_{h}^{n}\right)}{k}=c_{h}^{n}\left(K_{2}^{h}\right)^{\prime}\left(\xi_{h}^{n+1}\right) \frac{\phi_{h}^{n+1}-\phi_{h}^{n}}{k},
$$

where $\xi_{h}^{n+1} \in\left(\min \left\{\phi_{h}^{n}, \phi_{h}^{n+1}\right\}, \max \left\{\phi_{h}^{n}, \phi_{h}^{n+1}\right\}\right)$. Therefore,

$$
\frac{\mathrm{d}}{\mathrm{d} t} \widetilde{\varphi}_{h, k}=K_{2}^{h}\left(\phi_{h, k}\right) \frac{\mathrm{d}}{\mathrm{d} t} \widetilde{c}_{h, k}+\left(K_{2}^{h}\right)^{\prime}\left(\xi_{h, k}\right) \widehat{c}_{h, k} \frac{\mathrm{d}}{\mathrm{d} t} \widetilde{\phi}_{h, k} .
$$

We know that $\left\{K_{2}^{h}\left(\phi_{h, k}\right) \frac{\mathrm{d}}{\mathrm{d} t} \widetilde{c}_{h, k}\right\}_{h, k}$ is bounded in $L^{2}\left(0, T ;\left(W^{1, s}(\Omega)\right)^{\prime}\right)$ with $s>3$ (owing to $\left\{K_{2}^{h}\left(\phi_{h, k}\right)\right\}_{h, k}$ is bounded in $L^{\infty}\left(0, T ; H^{1}(\Omega)\right)$ and $\left.(4.2)\right)$ and $\left\{\left(K_{2}^{h}\right)^{\prime}\left(\xi_{h, k}\right) \widehat{c}_{h, k} \frac{\mathrm{d}}{\mathrm{d} t} \widetilde{\phi}_{h, k}\right\}_{h, k}$ is bounded in $L^{2}\left(0, T ; L^{1}(\Omega)\right)$ (owing to $\left\{\left(K_{2}^{h}\right)^{\prime}\left(\xi_{h, k}\right)\right\}_{h, k}$ is bounded in $L^{\infty}(Q),(3.12)$ and $\left.(3.16)\right)$; hence $\left\{\frac{\mathrm{d}}{\mathrm{d} t} \widetilde{\varphi}_{h, k}\right\}_{h, k}$ is bounded in $L^{1}\left(0, T ;\left(W^{1, s}(\Omega)\right)^{\prime}\right)$, with $s>3$. On the other hand, by using (3.12), (3.15) and the estimate of $\left\{\phi_{h, k}\right\}_{h, k}$ in $L^{4}\left(0, T ; W^{1,4}\right)$ given in (3.20), it is not hard to check that $\left\{\widetilde{\varphi}_{h, k}\right\}_{h, k}$ is bounded in $L^{2}\left(0, T ; W^{1,6 / 5}(\Omega)\right.$ ) (by assuming $K_{2}\left(\phi_{h}^{0}\right) c_{h}^{0}$ is bounded in $W^{1,6 / 5}(\Omega)$, which can be obtained as a consequence of inverse estimates and a constraint between $h$ and $k$ ).

Therefore, by a compactness result [13], there exists $\chi \in L^{2}\left(0, T ; L^{p}(\Omega)\right)$ with $p<2$, such that

$$
\widetilde{\varphi}_{h, k} \rightarrow \chi \text { in } L^{2}\left(0, T ; L^{p}(\Omega)\right), \quad \text { as }(h, k) \rightarrow 0 .
$$


In turn, $\varphi_{h, k}:=K_{2}^{h}\left(\phi_{h, k}\right) c_{h, k}$ tends strongly to $\chi$ in $L^{2}\left(0, T ; L^{3 / 2}(\Omega)\right)$, since the difference $R_{h, k}:=\widetilde{\varphi}_{h, k}(t)-$ $\varphi_{h, k}(t)$ tends to zero strongly in $L^{2}\left(0, T ; L^{3 / 2}(\Omega)\right)$. Indeed, we may write for $t \in\left(t_{n}, t_{n+1}\right)$

$$
R_{h, k}(t):=\frac{t-t_{n+1}}{k}\left(\left(K_{2}^{h}\right)^{\prime}\left(\xi_{h}^{n+1}\right)\left(\phi_{h}^{n+1}-\phi_{h}^{n}\right) c_{h}^{n+1}+K_{2}^{h}\left(\phi_{h}^{n}\right)\left(c_{h}^{n+1}-c_{h}^{n}\right)\right),
$$

with $\xi_{h}^{n+1}$ being as before. It thus follows that

$$
\begin{aligned}
\left\|R_{h, k}\right\|_{L^{2}\left(0, T ; L^{3 / 2}(\Omega)\right)} & \leq\left(C k \sum_{n=0}^{N-1}\left\|c_{h}^{n+1}-c_{h}^{n}\right\|_{L^{2}(\Omega)}^{2}\right)^{1 / 2}+\left(C k \sum_{n=0}^{N-1}\left\|\phi_{h}^{n+1}-\phi_{h}^{n+1}\right\|_{H^{1}(\Omega)}^{2}\right)^{1 / 2} \\
& \leq C k^{1 / 2} \rightarrow 0,
\end{aligned}
$$

due to estimates $(\boldsymbol{i i})$ and (viii) of Lemma 3.1. Then, as announced we have

$$
\varphi_{h, k} \rightarrow \chi \text { in } L^{2}\left(0, T ; L^{3 / 2}(\Omega)\right), \quad \text { as }(h, k) \rightarrow 0 .
$$

To identify $\chi=K_{2}(\phi) c$, we see that since $K_{2}$ is a globally Lipschitz continuous function on $\mathbb{R}$ and $\phi_{h, k}$ converges to $\phi$ in $L^{2}\left(0 ; T ; H^{1}(\Omega)\right)$, then (see [9], Thm. 16.7)

$$
K_{2}\left(\phi_{h, k}\right) \rightarrow K_{2}(\phi) \quad \text { strongly in } L^{2}\left(0, T ; H^{1}(\Omega)\right) .
$$

Thus, $K_{2}^{h}\left(\phi_{h, k}\right) c_{h, k}$ converges weakly to $K_{2}(\phi) c$ in $L^{2}\left(0, T ; L^{3 / 2}(\Omega)\right)$ by taking into account that $g_{2}(h) c_{h, k}$ converges strongly to 0 in $L^{\infty}\left(0, T ; L^{2}(\Omega)\right)$ as $(h, k) \rightarrow 0$. Therefore, we can identify $\chi=K_{2}(\phi) c$ and

$$
\varphi_{h, k}=K_{2}^{h}\left(\phi_{h, k}\right) c_{h, k} \rightarrow K_{2}(\phi) c \quad \text { strongly in } L^{2}\left(0, T ; L^{3 / 2}(\Omega)\right) \text { as }(h, k) \rightarrow 0 .
$$

As a consequence, by using estimate (viii) of Lemma 3.1, we have

$$
K_{2}^{h}\left(\phi_{h, k}\right) \widehat{c}_{h, k} \rightarrow K_{2}(\phi) c \text { strongly in } L^{2}\left(0, T ; L^{3 / 2}(\Omega)\right) \text {, as }(h, k) \rightarrow 0 .
$$

From (4.10), we get $K_{2}\left(\phi_{h, k}(\boldsymbol{x}, t)\right) \rightarrow K_{2}(\phi(\boldsymbol{x}, t))$ a.e. $(\boldsymbol{x}, t) \in Q$. In particular, one has

$$
K_{2}^{h}\left(\phi_{h, k}(\boldsymbol{x}, t)\right) \rightarrow K_{2}(\phi(\boldsymbol{x}, t)) \text { a.e. }(\boldsymbol{x}, t) \in Q .
$$

If we define $\widetilde{Q}=\left\{(\boldsymbol{x}, t) \in Q: K_{2}(\phi(\boldsymbol{x}, t))>0\right\}$, since $K_{2}^{h}\left(\phi_{h, k}(\boldsymbol{x}, t)\right)>0$ for all $(\boldsymbol{x}, t)$, it is easy to prove that

$$
\widehat{c}_{h, k}(\boldsymbol{x}, t) \rightarrow c(\boldsymbol{x}, t) \text { a.e. }(\boldsymbol{x}, t) \in \widetilde{Q}
$$

from (4.11) and the pointwise convergence (4.12).

Once we have achieved the pointwise convergence of $\widehat{c}_{h, k}$ to the limit $c$, let us see the pointwise convergence

$$
\left[P_{0} \widehat{c}_{h, k}(\boldsymbol{x}, t)\right]_{T} \rightarrow T_{0}^{1} c(\boldsymbol{x}, t) \text { a.e. }(\boldsymbol{x}, t) \in \widetilde{Q} .
$$

Indeed, this convergence follows from the inequality

$$
\left|\left[P_{0} \widehat{c}_{h, k}(\boldsymbol{x}, t)\right]_{T}-T_{0}^{1} c(\boldsymbol{x}, t)\right|=\left|T_{0}^{1}\left(P_{0} \widehat{c}_{h, k}(\boldsymbol{x}, t)\right)-T_{0}^{1} c(\boldsymbol{x}, t)\right| \leq\left|P_{0} \widehat{c}_{h, k}(\boldsymbol{x}, t)-c(\boldsymbol{x}, t)\right| \text { a.e. }(\boldsymbol{x}, t) \in Q,
$$

the triangular inequality and the pointwise convergence as a consequence of (4.5).

Finally,

$$
K_{2}^{h}\left(\phi_{h, k}(\boldsymbol{x}, t)\right)\left[P_{0} \widehat{c}_{h, k}\right]_{T} \rightarrow K_{2}(\phi(\boldsymbol{x}, t)) T_{0}^{1} c(\boldsymbol{x}, t) \text { a.e. }(\boldsymbol{x}, t) \in Q
$$


holds from (4.12), (4.14), and $K_{2}^{h}\left(\phi_{h, k}(\boldsymbol{x}, t)\right)$ converges to 0 a.e. in $Q \backslash \widetilde{Q}$. In particular,

$$
K_{2}^{h}\left(\phi_{h, k}(\boldsymbol{x}, t)\right)\left[P_{0} \widehat{c}_{h, k}\right]_{T}^{2} \rightarrow K_{2}(\phi(\boldsymbol{x}, t)) T_{0}^{1} c(\boldsymbol{x}, t)^{2} \text { a.e. }(\boldsymbol{x}, t) \in Q .
$$

Then, (4.7) holds as a consequence of the dominated convergence theorem, and the proof of Proposition 4.3 is finished.

Remark 4.4. In the case of a nondegenerate solute diffusivity $K_{2}$ we may prove firstly that $\widehat{c}_{h, k} \rightarrow c$ strongly in $L^{2}\left(0, T ; L^{2}(\Omega)\right)$ by a compactness result, then this convergence is extended to $P_{0} \widehat{c}_{h, k} \rightarrow c$ strongly in $L^{2}\left(0, T ; L^{2}(\Omega)\right)$ from Proposition 4.3 , and finally we may establish $\left[\widehat{P}_{0} c_{h, k}\right]_{T} \rightarrow T_{0}^{1} c$ strongly in $L^{2}\left(0, T ; L^{2}(\Omega)\right)$ as an application of the dominated convergence theorem.

Now, we want to identify $J_{1}=\nabla\left(K_{1}(\phi) \theta\right)-\theta \nabla K_{1}(\phi)$ (and $\left.J_{2}=\nabla\left(K_{2}(\phi) c\right)-c \nabla K_{2}(\phi)\right)$. Indeed, analogue to (4.10) we have

$$
K_{1}\left(\phi_{h, k}\right) \rightarrow K_{1}(\phi) \quad \text { strongly in } L^{2}\left(0, T ; H^{1}(\Omega)\right) .
$$

On the other hand, using the fact that $\theta_{h, k} \rightarrow \theta$ weakly* in $L^{\infty}\left(0, T ; L^{2}(\Omega)\right)$ and (4.15), the following weak convergences hold:

$$
\begin{gathered}
\theta_{h, k} \nabla K_{1}\left(\phi_{h, k}\right) \rightarrow \theta \nabla K_{1}(\phi) \quad \text { weakly in } L^{2}\left(0 ; T ; L^{1}(\Omega)\right), \\
\theta_{h, k} K_{1}\left(\phi_{h, k}\right) \rightarrow \theta K_{1}(\phi) \quad \text { weakly in } L^{2}\left(0 ; T ; L^{3 / 2}(\Omega)\right) .
\end{gathered}
$$

Now, using the regularity $K_{1}\left(\phi_{h, k}\right) \in L^{\infty}\left(0, T ; H^{1}(\Omega)\right)$ and $\theta_{h, k} \in L^{2}\left(0, T ; H^{1}(\Omega)\right)$ (because of $0<g_{1}(h) \leq$ $\left.K_{1}^{h}(\cdot)\right)$ and the Sobolev product $\|\varphi \psi\|_{W^{1,3 / 2}(\Omega)} \leq C\|\varphi\|_{H^{1}(\Omega)}\|\psi\|_{H^{1}(\Omega)}$ for all $\varphi, \psi \in H^{1}(\Omega)$, we get

$$
K_{1}\left(\phi_{h, k}\right) \theta_{h, k} \in L^{2}\left(0, T ; W^{1,3 / 2}(\Omega)\right)
$$

and, in particular,

$$
\nabla\left(K_{1}\left(\phi_{h, k}\right) \theta_{h, k}\right)=K_{1}\left(\phi_{h, k}\right) \nabla \theta_{h, k}+\theta_{h, k} \nabla K_{1}\left(\phi_{h, k}\right) .
$$

Therefore, in view of the convergences (4.16), (4.17) and the identity (4.18), one arrives at

$$
K_{1}\left(\phi_{h, k}\right) \nabla \theta_{h, k} \rightarrow \nabla\left(K_{1}(\phi) \theta\right)-\theta \nabla K_{1}(\phi) \quad \text { in } L^{2}\left(0, T ; W^{-1,3 / 2-\varepsilon}(\Omega)\right)
$$

with $1 / 2>\varepsilon>0$.

Next, recalling the definition of $K_{1}^{h}=g_{1}(h)+K_{1}$, we write

$$
K_{1}^{h}\left(\phi_{h, k}\right) \nabla \theta_{h, k}=g_{1}(h) \nabla \theta_{h, k}+K_{1}\left(\phi_{h, k}\right) \nabla \theta_{h, k} .
$$

Now, taking into account that $\left\|g_{1}(h)^{1 / 2} \nabla \theta_{h, k}\right\|_{L^{2}(Q)} \leq\left\|\sqrt{K_{1}^{h}\left(\phi_{h, k}\right)} \nabla \theta_{h, k}\right\|_{L^{2}(Q)} \leq C$ with $C>0$ independent of $(k, h)$, one obtains

$$
g_{1}(h) \nabla \theta_{h, k} \rightarrow 0 \quad \text { in } L^{2}(Q)
$$

that jointly with (4.19) and (4.20) gives us

$$
K_{1}^{h}\left(\phi_{h, k}\right) \nabla \theta_{h, k} \rightarrow \nabla\left(K_{1}(\phi) \theta\right)-\theta \nabla K_{1}(\phi) \quad \text { in } L^{2}\left(0, T ; W^{-1,3 / 2-\varepsilon}(\Omega)\right) .
$$

Finally, this convergence and the weak convergence to $J_{1}$ given in Lemma 3.6 conclude the identification $J_{1}=\nabla\left(K_{1}(\phi) \theta\right)-\theta \nabla K_{1}(\phi)$. 


\section{PASSING TO THE LIMIT}

In order to pass to the limit in the discrete concentration equation, we will use the following result, which is easy to prove because equation (5.1) satisfies the maximum principle:

Lemma 5.1. The following two systems are equivalent:

$$
c_{t}=\nabla \cdot\left(K_{2}(\phi)\left[\nabla c+M T_{0}^{1} c\left(1-T_{0}^{1} c\right) \nabla \phi\right]\right) \quad \text { in } Q
$$

and

$$
0 \leq c \leq 1, \quad c_{t}=\nabla \cdot\left(K_{2}(\phi)[\nabla c+M c(1-c) \nabla \phi]\right) \quad \text { in } Q .
$$

To pass to the limit in scheme $(2.1)-(2.3)$, we rewrite the scheme as follows: Taking $x_{h}=\eta_{h}^{n+1} \in X_{h}$ a suitable approximation at time $t_{n+1}$ of any function $\eta \in C^{0}\left([0, T] ; C_{c}^{\infty}(\Omega)\right)$ such that $\eta(T)=0\left(\right.$ clearly $\left.\eta_{h}^{N}=0\right)$ as a test function in (2.1), (2.2) and (2.3), multiplying by $k$, summing over $n$ and denoting the function $\eta_{h, k}$ similarly to Definition 3.5 , one arrives at

$$
\begin{aligned}
& \left\{\begin{array}{l}
\alpha \varepsilon^{2} \int_{0}^{T}\left(\frac{\mathrm{d}}{\mathrm{d} t} \widetilde{\phi}_{h, k}, \eta_{h, k}\right)+\varepsilon^{2} \int_{0}^{T}\left(\nabla \phi_{h, k}, \nabla \eta_{h, k}\right)+\varepsilon^{2} \int_{0}^{T}\left(\phi_{h, k}-\widehat{\phi}_{h, k}, \eta_{h, k}\right) \\
+\frac{1}{2} \int_{0}^{T}\left(\left(\phi_{h, k}\right)^{3}-\widehat{\phi}_{h, k}, \eta_{h, k}\right)-\beta \int_{0}^{T}\left(\widehat{\theta}_{h, k}-\theta_{A} \widehat{c}_{h, k}-\theta_{B}\left(1-\widehat{c}_{h, k}\right), \eta_{h, k}\right)=0,
\end{array}\right. \\
& C_{V} \int_{0}^{T}\left(\frac{\mathrm{d}}{\mathrm{d} t} \widetilde{\theta}_{h, k}, \eta_{h, k}\right)+\frac{l}{2} \int_{0}^{T}\left(\frac{\mathrm{d}}{\mathrm{d} t} \widetilde{\phi}_{h, k}, \eta_{h, k}\right)+\int_{0}^{T}\left(K_{1}^{h}\left(\phi_{h, k}\right) \nabla \theta_{h, k}, \nabla \eta_{h, k}\right)=0 \\
& \left\{\begin{array}{l}
\int_{0}^{T}\left(\frac{\mathrm{d}}{\mathrm{d} t} \widetilde{c}_{h, k}, \eta_{h, k}\right)+\int_{0}^{T}\left(K_{2}^{h}\left(\phi_{h, k}\right) \nabla c_{h, k}, \nabla \eta_{h, k}\right) \\
+M \int_{0}^{T}\left(K_{2}^{h}\left(\phi_{h, k}\right)\left[P_{0} \widehat{c}_{h, k}\right]_{T}\left(1-\left[P_{0} \widehat{c}_{h, k}\right]_{T}\right) \nabla \widehat{\phi}_{h, k}, \nabla \eta_{h, k}\right)=0 .
\end{array}\right.
\end{aligned}
$$

By applying all the convergences already obtained, there are no additional difficulties in passing to the limit obtaining that $(\phi, \theta, c)$ is a weak solution of (1.1). In particular, taking $(h, k) \rightarrow 0$ in the discrete equation for the concentration $c$ and using (4.7), we arrive at the limit equation (5.1); hence $0 \leq c \leq 1$ and $T_{0}^{1} c=c$. Finally, the discrete phase-field equation is verified pointwise in $Q$ thanks to the strong regularity of $\phi$. The proof of Theorem 1.3 is finished.

Remark 5.2. As mentioned in Remark 3.2, taking $\left[P_{0} c_{h}^{n}\right]$ instead of $c_{h}^{n}$ in the discrete equation for the phase field variable (2.1) provides better stability estimates with respect to $\varepsilon$. Nevertheless we find that there is a limit function $\vartheta \in L^{\infty}\left(0, T ; L^{2}(\Omega)\right)$ such that $\left[P_{0} c_{h}^{n}\right]$ tends to $\vartheta$ weakly* in $L^{\infty}\left(0, T ; L^{2}(\Omega)\right)$, but it is not clear how to identify $\vartheta$ with the limit function $c$. By using (4.14) and that $0 \leq c \leq 1$ a.e. $Q$ (owing to the limit in (5.2) can be taken as before), we can only deduce that $\vartheta=c$ a.e. in $\widetilde{Q}, \widetilde{Q}$ being defined in the proof of Proposition 4.3 .

\section{A COnditionally stable, COnVERGEnt Linear SCHEMe}

In this section we study a more explicit scheme, where the nonlinear discrete approximation $(2.1)$ of $(1.1)_{1}$ is considered completely in the previous step time, resulting a linear (and decoupled) scheme. Contrary to the previous nonlinear scheme, now to obtain stability we will impose a constraint on the discrete parameters.

Recall the definition of the Ginzburg-Landau function $f(\phi)=\frac{1}{2 \varepsilon^{2}}\left(\phi^{2}-1\right) \phi$ associated to the potential function $F(\phi)=\frac{1}{8 \varepsilon^{2}}\left(\phi^{2}-1\right)^{2}$. We propose the following linear scheme:

Initialization: Let $\left(\phi_{h}^{0}, \theta_{h}^{0}, c_{h}^{0}\right) \in X_{h} \times X_{h} \times X_{h}$ be suitable approximations of $\left(\phi_{0}, \theta_{0}, c_{0}\right)$.

Step $n+1$ : Given $\left(\phi_{h}^{n}, \theta_{h}^{n}, c_{h}^{n}\right) \in X_{h} \times X_{h} \times X_{h}$. 
Find $\phi_{h}^{n+1} \in X_{h}$ as a solution of the problem:

$$
\begin{aligned}
\alpha\left(\frac{\phi_{h}^{n+1}-\phi_{h}^{n}}{k}, x_{h}\right)+\left(\nabla \phi_{h}^{n+1}, \nabla x_{h}\right)+\left(\phi_{h}^{n+1}, x_{h}\right) & =-\left(f\left(\phi_{h}^{n}\right), x_{h}\right)+\left(\phi_{h}^{n}, x_{h}\right) \\
& +\frac{\beta}{\varepsilon^{2}}\left(\theta_{h}^{n}-\theta_{A} c_{h}^{n}-\theta_{B}\left(1-c_{h}^{n}\right), x_{h}\right), \quad \forall x_{h} \in X_{h} .
\end{aligned}
$$

Find $\theta_{h}^{n+1} \in X_{h}$ and $c_{h}^{n+1} \in X_{h}$ as solutions of the decoupled variational problems:

$$
\begin{gathered}
C_{V}\left(\frac{\theta_{h}^{n+1}-\theta_{h}^{n}}{k}, x_{h}\right)+\left(K_{1}^{h}\left(\phi_{h}^{n+1}\right) \nabla \theta_{h}^{n+1}, \nabla x_{h}\right)=-\frac{l}{2}\left(\frac{\phi_{h}^{n+1}-\phi_{h}^{n}}{k}, x_{h}\right), \quad \forall x_{h} \in X_{h}, \\
\left\{\begin{array}{l}
\left(\frac{c_{h}^{n+1}-c_{h}^{n}}{k}, x_{h}\right)+\left(K_{2}^{h}\left(\phi_{h}^{n+1}\right) \nabla c_{h}^{n+1}, \nabla x_{h}\right) \\
=-M\left(K_{2}^{h}\left(\phi_{h}^{n+1}\right)\left[P_{0} c_{h}^{n}\right]_{T}\left(1-\left[P_{0} c_{h}^{n}\right]_{T}\right) \nabla \phi_{h}^{n}, \nabla x_{h}\right), \quad \forall x_{h} \in X_{h} .
\end{array}\right.
\end{gathered}
$$

The conditional stability of scheme (6.1)-(6.3) will be obtained by induction on the time step $n$. First of all, we establish the following result which provides a basic recursive inequality.

Lemma 6.1. Assume the constraint:

$$
\lim _{(h, k) \rightarrow 0} k / h=0 .
$$

If there exists a constant $C_{d}>0$ (independent of $h, k$ and $n$, but dependent on $\varepsilon$ ) such that

$$
\left\|\phi_{h}^{n}\right\|_{H^{1}(\Omega)}^{2}+\frac{2 C_{V} \beta}{l \varepsilon^{2}}\left|\theta_{h}^{n}\right|^{2}+\left|c_{h}^{n}\right|^{2}+1 \leq C_{d},
$$

then the following inequalities hold for $(h, k)$ sufficiently small (independent of $n$ ),

$$
\left\{\begin{array}{l}
\left(\left\|\phi_{h}^{n+1}\right\|_{H^{1}(\Omega)}^{2}-\left\|\phi_{h}^{n}\right\|_{H^{1}(\Omega)}^{2}+\left\|\phi_{h}^{n+1}-\phi_{h}^{n}\right\|_{H^{1}(\Omega)}^{2}\right) \\
+\frac{2 C_{V} \beta}{l \varepsilon^{2}}\left(\left|\theta_{h}^{n+1}\right|^{2}-\left|\theta_{h}^{n}\right|^{2}+\frac{1}{2}\left|\theta_{h}^{n+1}-\theta_{h}^{n}\right|^{2}\right) \\
+2 \int_{\Omega}\left(F\left(\phi_{h}^{n+1}\right)-F\left(\phi_{h}^{n}\right)+\frac{1}{8 \varepsilon^{2}}\left(\left(\phi_{h}^{n+1}\right)^{2}-\left(\phi_{h}^{n}\right)^{2}\right)^{2}\right) \\
+\frac{\alpha}{2} k\left|\frac{\phi_{h}^{n+1}-\phi_{h}^{n}}{k}\right|^{2}+\frac{2 \beta}{l \varepsilon^{2}} k\left|\sqrt{K_{1}^{h}\left(\phi_{h}^{n+1}\right)} \nabla \theta_{h}^{n+1}\right|^{2} \\
\leq R_{1} k\left|\phi_{h}^{n}\right|^{2}+R_{2} \frac{\beta^{2}}{\varepsilon^{4}} k\left(\left|c_{h}^{n}\right|^{2}+1\right),
\end{array}\right.
$$

where $R_{1}$ and $R_{2}$ are positive constants independent of $h, k, n$, and $\varepsilon$.

Proof. Firstly, we consider $x_{h}=2 k \frac{\phi_{h}^{n+1}-\phi_{h}^{n}}{k}$ in (6.1) and bound the right-hand side

$$
\begin{aligned}
\frac{3}{2} \alpha k\left|\frac{\phi_{h}^{n+1}-\phi_{h}^{n}}{k}\right|^{2}+ & \left(\left\|\phi_{h}^{n+1}\right\|_{H^{1}(\Omega)}^{2}-\left\|\phi_{h}^{n}\right\|_{H^{1}(\Omega)}^{2}+\left\|\phi_{h}^{n+1}-\phi_{h}^{n}\right\|_{H^{1}(\Omega)}^{2}\right) \\
& +2\left(f\left(\phi_{h}^{n}\right), \phi_{h}^{n+1}-\phi_{h}^{n}\right) \leq \frac{2 \beta}{\varepsilon^{2}} k\left(\theta_{h}^{n}, \frac{\phi_{h}^{n+1}-\phi_{h}^{n}}{k}\right)+\frac{C}{\alpha} k\left|\phi_{h}^{n}\right|^{2}+C \frac{\beta^{2}}{\alpha \varepsilon^{4}} k\left(\left|c_{h}^{n}\right|^{2}+1\right) .
\end{aligned}
$$


Now, we handle the last term on the left-hand side of (6.7) as follows:

$$
2\left(\phi_{h}^{n+1}-\phi_{h}^{n}, f\left(\phi_{h}^{n}\right)\right)=\frac{1}{\varepsilon^{2}}\left(\phi_{h}^{n+1}-\phi_{h}^{n},\left(\left(\phi_{h}^{n+1}\right)^{2}-1\right) \phi_{h}^{n}\right)+\frac{1}{\varepsilon^{2}}\left(\phi_{h}^{n+1}-\phi_{h}^{n},\left(\left(\phi_{h}^{n}\right)^{2}-\left(\phi_{h}^{n+1}\right)^{2}\right) \phi_{h}^{n}\right):=I_{1}-I_{2} .
$$

Next, we continue rewriting $I_{1}$ as follows:

$$
\begin{aligned}
I_{1}= & \frac{1}{2 \varepsilon^{2}} \int_{\Omega}\left(\left(\phi_{h}^{n+1}\right)^{2}-1\right)\left(\left(\phi_{h}^{n+1}\right)^{2}-\left(\phi_{h}^{n}\right)^{2}-\left(\phi_{h}^{n+1}-\phi_{h}^{n}\right)^{2}\right) \\
= & \frac{1}{4 \varepsilon^{2}} \int_{\Omega}\left(\left(\left(\phi_{h}^{n+1}\right)^{2}-1\right)^{2}-\left(\left(\phi_{h}^{n}\right)^{2}-1\right)^{2}+\left(\left(\phi_{h}^{n+1}\right)^{2}-\left(\phi_{h}^{n}\right)^{2}\right)^{2}\right) \\
& +\frac{k^{2}}{2 \varepsilon^{2}} \int_{\Omega}\left(1-\left(\phi_{h}^{n+1}\right)^{2}\right)\left|\frac{\phi_{h}^{n+1}-\phi_{h}^{n}}{k}\right|^{2} .
\end{aligned}
$$

The term $I_{2}$ is bounded as

$$
I_{2} \leq C \frac{1}{\varepsilon^{2}} k^{2}\left\|\phi_{h}^{n}\right\|_{L^{\infty}(\Omega)}^{2}\left|\frac{\phi_{h}^{n+1}-\phi_{h}^{n}}{k}\right|^{2}+\frac{1}{8 \varepsilon^{2}} \int_{\Omega}\left(\left(\phi_{h}^{n+1}\right)^{2}-\left(\phi_{h}^{n}\right)^{2}\right)^{2} .
$$

Therefore, we get from (6.7) and the previous computations

$$
\begin{aligned}
& \frac{3}{2} \alpha k\left|\frac{\phi_{h}^{n+1}-\phi_{h}^{n}}{k}\right|^{2}+\left(\left\|\phi_{h}^{n+1}\right\|_{H^{1}(\Omega)}^{2}-\left\|\phi_{h}^{n}\right\|_{H^{1}(\Omega)}^{2}+\left\|\phi_{h}^{n+1}-\phi_{h}^{n}\right\|_{H^{1}(\Omega)}^{2}\right) \\
& \quad+2 \int_{\Omega}\left(F\left(\phi_{h}^{n+1}\right)-F\left(\phi_{h}^{n}\right)+\frac{1}{8 \varepsilon^{2}}\left(\left(\phi_{h}^{n+1}\right)^{2}-\left(\phi_{h}^{n}\right)^{2}\right)^{2}\right)+\frac{k^{2}}{2 \varepsilon^{2}}\left|\frac{\phi_{h}^{n+1}-\phi_{h}^{n}}{k}\right|^{2} \\
& \leq \frac{2 \beta}{\varepsilon^{2}} k\left(\theta_{h}^{n}, \frac{\phi_{h}^{n+1}-\phi_{h}^{n}}{k}\right)+\frac{C}{\alpha} k\left|\phi_{h}^{n}\right|^{2}+C \frac{\beta^{2}}{\varepsilon^{4} \alpha} k\left(\left|c_{h}^{n}\right|^{2}+1\right) \\
& \quad+C \frac{1}{\varepsilon^{2} \alpha} k\left(\left\|\phi_{h}^{n}\right\|_{L^{\infty}(\Omega)}^{2}+\left\|\phi_{h}^{n+1}\right\|_{L^{\infty}(\Omega)}^{2}\right) k\left|\frac{\phi_{h}^{n+1}-\phi_{h}^{n}}{k}\right|^{2} \\
& \leq \\
& \frac{2 \beta}{\varepsilon^{2}} k\left(\theta_{h}^{n}, \frac{\phi_{h}^{n+1}-\phi_{h}^{n}}{k}\right)+\frac{C}{\alpha} k\left|\phi_{h}^{n}\right|^{2}+C \frac{\beta^{2}}{\varepsilon^{4}} k\left(\left|c_{h}^{n}\right|^{2}+1\right) \\
& \quad+C \frac{1}{\varepsilon^{2}} \frac{k}{h}\left(\left\|\phi_{h}^{n}\right\|_{H^{1}(\Omega)}^{2}+\left\|\phi_{h}^{n+1}\right\|_{H^{1}(\Omega)}^{2}\right) k\left|\frac{\phi_{h}^{n+1}-\phi_{h}^{n}}{k}\right|^{2},
\end{aligned}
$$

where in the last line the inverse estimate $\left\|x_{h}\right\|_{L^{\infty}(\Omega)} \leq C h^{-1 / 2}\left\|x_{h}\right\|_{H^{1}(\Omega)}$ has been used.

Now we are looking for the bound $\left\|\phi_{h}^{n+1}\right\|_{H^{1}(\Omega)} \leq C_{1}$ where $C_{1}>0$ depends on the constant $C_{d}$ of hypothesis (6.4) but it will be independent of $n$. It will be carried out by bounding $\left\|\phi_{h}^{n+1}\right\|_{H^{1}(\Omega)}$ in terms of $\left\|\phi_{h}^{n}\right\|_{H^{1}(\Omega)}$, $\left|\theta_{h}^{n}\right|$ and $\left|c_{h}^{n}\right|$ and using hypothesis (6.4). Indeed, taking again $x_{h}=2 k \frac{\phi_{h}^{n+1}-\phi_{h}^{n}}{k}$ as a test function in (6.1), 
but now bounding directly the term depending on $f\left(\phi_{h}^{n}\right)$ on the right-hand side, we get

$$
\begin{aligned}
\alpha k\left|\frac{\phi_{h}^{n+1}-\phi_{h}^{n}}{k}\right|^{2}+ & \left(\left\|\phi_{h}^{n+1}\right\|_{H^{1}(\Omega)}^{2}-\left\|\phi_{h}^{n}\right\|_{H^{1}(\Omega)}^{2}+\left\|\phi_{h}^{n+1}-\phi_{h}^{n}\right\|_{H^{1}(\Omega)}^{2}\right) \leq C \frac{\beta^{2}}{\varepsilon^{4} \alpha} k\left|\theta_{h}^{n}\right|^{2} \\
& +\frac{C}{\alpha} k\left|\phi_{h}^{n}\right|^{2}+C \frac{\beta^{2}}{\varepsilon^{4} \alpha} k\left(\left|c_{h}^{n}\right|^{2}+1\right)+C k\left|f\left(\phi_{h}^{n}\right)\right|^{2} \\
\leq & C \frac{\beta^{2}}{\varepsilon^{4} \alpha} k\left|\theta_{h}^{n}\right|^{2}+\frac{C}{\alpha} k\left|\phi_{h}^{n}\right|^{2}+C \frac{\beta^{2}}{\varepsilon^{4} \alpha} k\left(\left|c_{h}^{n}\right|^{2}+1\right) \\
& +C \frac{1}{\varepsilon^{4}} k\left\|\phi_{h}^{n}\right\|_{H^{1}(\Omega)}^{6}+C \frac{1}{\varepsilon^{4}} k\left\|\phi_{h}^{n}\right\|_{H^{1}(\Omega)}^{2} .
\end{aligned}
$$

In particular, by using hypothesis (6.4), the previous inequality says us

$$
\left\|\phi_{h}^{n+1}\right\|_{H^{1}(\Omega)}^{2} \leq\left\|\phi_{h}^{n}\right\|_{H^{1}(\Omega)}^{2}+C k\left(\frac{C_{d} \beta}{\varepsilon^{2}}+C_{d}+\frac{C_{d} \beta^{2}}{\varepsilon^{4}}+\frac{C_{d}^{3}}{\varepsilon^{4}}+\frac{C_{d}}{\varepsilon^{4}}\right) \leq\left\|\phi_{h}^{n}\right\|_{H^{1}(\Omega)}^{2}+C_{1}(\varepsilon) k
$$

with $C_{1}(\varepsilon)$ independent of $h, k$ and $n$.

Thus, by using the previous estimate in (6.9) and again hypothesis (6.4), we get

$$
\begin{aligned}
& \frac{3}{2} \alpha k\left|\frac{\phi_{h}^{n+1}-\phi_{h}^{n}}{k}\right|^{2}+\left(\left\|\phi_{h}^{n+1}\right\|_{H^{1}(\Omega)}^{2}-\left\|\phi_{h}^{n}\right\|_{H^{1}(\Omega)}^{2}+\left\|\phi_{h}^{n+1}-\phi_{h}^{n}\right\|_{H^{1}(\Omega)}^{2}\right) \\
& \quad+2 \int_{\Omega}\left(F\left(\phi_{h}^{n+1}\right)-F\left(\phi_{h}^{n}\right)+\frac{1}{8 \varepsilon^{2}}\left(\left(\phi_{h}^{n+1}\right)^{2}-\left(\phi_{h}^{n}\right)^{2}\right)^{2}\right) \\
& \leq \frac{2 \beta}{\varepsilon^{2}} k\left(\theta_{h}^{n}, \frac{\phi_{h}^{n+1}-\phi_{h}^{n}}{k}\right)+\frac{C}{\alpha} k\left|\phi_{h}^{n}\right|^{2}+C \frac{\beta^{2}}{\alpha \varepsilon^{4}} k\left(\left|c_{h}^{n}\right|^{2}+1\right)+C \frac{k}{h} \frac{1}{\varepsilon^{2}}\left(C_{d}+C_{1}(\varepsilon) k\right) k\left|\frac{\phi_{h}^{n+1}-\phi_{h}^{n}}{k}\right|^{2}
\end{aligned}
$$

By taking into account the constraint $(S)$, in particular $\lim _{(h, k) \rightarrow 0} \frac{k}{h} \frac{1}{\varepsilon^{2}}\left(C_{d}+C_{1}(\varepsilon) k\right)=0$, so for any $(h, k)$ small enough such that $C \frac{k}{h} \frac{1}{\varepsilon^{2}}\left(C_{d}+C_{1}(\varepsilon) k\right) \leq \frac{1}{2} \alpha$, the last term on the right-hand side can be absorbed, and remains

$$
\begin{aligned}
\alpha k\left|\frac{\phi_{h}^{n+1}-\phi_{h}^{n}}{k}\right|^{2} & +\left(\left\|\phi_{h}^{n+1}\right\|_{H^{1}(\Omega)}^{2}-\left\|\phi_{h}^{n}\right\|_{H^{1}(\Omega)}^{2}+\left\|\phi_{h}^{n+1}-\phi_{h}^{n}\right\|_{H^{1}(\Omega)}^{2}\right)+2 \int_{\Omega}\left(F\left(\phi_{h}^{n+1}\right)-F\left(\phi_{h}^{n}\right)\right. \\
& \left.+\frac{1}{8 \varepsilon^{2}}\left(\left(\phi_{h}^{n+1}\right)^{2}-\left(\phi_{h}^{n}\right)^{2}\right)^{2}\right) \leq \frac{2 \beta}{\varepsilon^{2}} k\left(\theta_{h}^{n}, \frac{\phi_{h}^{n+1}-\phi_{h}^{n}}{k}\right)+\frac{C}{\alpha} k\left|\phi_{h}^{n}\right|^{2}+C \frac{\beta^{2}}{\alpha \varepsilon^{4}} k\left(\left|c_{h}^{n}\right|^{2}+1\right)
\end{aligned}
$$

On the other hand, take $x_{h}=\frac{4 \beta}{l \varepsilon^{2}} k \theta_{h}^{n+1}$ in (6.2) to arrive at inequality (3.5), that is

$$
\begin{aligned}
\frac{2 C_{V} \beta}{l \varepsilon^{2}}\left(\left|\theta_{h}^{n+1}\right|^{2}-\left|\theta_{h}^{n}\right|^{2}+\frac{1}{2}\left|\theta_{h}^{n+1}-\theta_{h}^{n}\right|^{2}\right)+\frac{4 \beta}{l \varepsilon^{2}} k\left|\sqrt{K_{1}^{h}\left(\phi_{h}^{n+1}\right)} \nabla \theta_{h}^{n+1}\right|^{2} \leq \\
\quad-\frac{2 \beta}{\varepsilon^{2}} k\left(\frac{\phi_{h}^{n+1}-\phi_{h}^{n}}{k}, \theta_{h}^{n}\right)+\frac{\alpha}{2} k\left|\frac{\phi_{h}^{n+1}-\phi_{h}^{n}}{k}\right|^{2} .
\end{aligned}
$$

Consequently, it suffices to add up (6.11) and (6.10) to get (6.5).

Finally, inequality (6.6) is easily obtained by testing (6.3) by $c_{h}^{n+1}$ and bounding adequately as in the proof of Lemma 3.1. 
On the other hand, we turn our attention to the initial bound (3.2) which in particular verifies hypothesis (6.4) imposed in Lemma 6.1. It is very important in order to guarantee a correct induction argument.

Now, we are in position to give the following stability result.

Lemma 6.2. Under the hypotheses of Lemma 6.1, the discrete solution of scheme (6.1)-(6.3) satisfies the following estimates:
(i) $\max _{0 \leq n \leq N}\left\|\phi_{h}^{n}\right\|_{H^{1}(\Omega)}^{2} \leq C$,
(ii) $\sum_{n=0}^{N-1}\left\|\phi_{h}^{n+1}-\phi_{h}^{n}\right\|_{H^{1}(\Omega)}^{2} \leq C$,
(iii) $k \sum_{n=0}^{N-1}\left|\frac{\phi_{h}^{n+1}-\phi_{h}^{n}}{k}\right|^{2} \leq C$,
(iv) $\max _{0 \leq n \leq N}\left|\theta_{h}^{n}\right|^{2} \leq C$
(v) $\sum_{n=0}^{N-1}\left|\theta_{h}^{n+1}-\theta_{h}^{n}\right|^{2} \leq C$,
(vi) $k \sum_{n=0}^{N-1}\left|\sqrt{K_{1}^{h}\left(\phi_{h}^{n+1}\right)} \nabla \theta_{h}^{n+1}\right|^{2} \leq C$,
(vii) $\max _{0 \leq n \leq N}\left|c_{h}^{n}\right|^{2} \leq C$
(viii) $\sum_{n=0}^{N-1}\left|c_{h}^{n+1}-c_{h}^{n}\right|^{2} \leq C$,
(ix) $k \sum_{n=0}^{N-1}\left|\sqrt{K_{2}^{h}\left(\phi_{h}^{n+1}\right)} \nabla c_{h}^{n+1}\right|^{2} \leq C$,

where $C>0$ is independent of $(h, k)$ and depends on the data $\left(\phi_{0}, \theta_{0}, c_{0}\right), \alpha, \beta$ and $\varepsilon$.

Proof. Obviously, if we let (6.5) and (6.6) hold for $n=0, \ldots, N-1$, we get all the statements of this lemma by adding (6.5) and (6.6) and applying the discrete Gronwall lemma. Therefore, it suffices to prove that (6.5) and (6.6) hold for $n=0, \ldots, N-1$.

Let us consider $C_{d}=\mathrm{e}^{\left(R_{1}+\frac{\beta^{2}}{\varepsilon^{4}} R_{2}\right) T} C_{2} / \varepsilon^{2}$ with $C_{2}>0$ given in (3.2) and $R_{1}, R_{2}$ given in Lemma 6.1. As the initial approximations hold hypothesis (6.4) for $n=0$, inequalities (6.5) and (6.6) are satisfied for $n=0$.

The final induction step can be easily seen by assuming that inequalities (6.5) and (6.6) hold for $l=0, \ldots, n-1$. Then, adding up (6.5) and (6.6) from 0 to $n-1$, one has

$$
\begin{aligned}
\frac{2 C_{V} \beta}{l \varepsilon^{2}}\left|\theta_{h}^{n}\right|^{2}+\left|c_{h}^{n}\right|^{2}+1+\left\|\phi_{h}^{n}\right\|_{H^{1}(\Omega)}^{2}+2 \int_{\Omega} F\left(\phi_{h}^{n}\right) \leq & \frac{2 C_{V} \beta}{l \varepsilon^{2}}\left|\theta_{h}^{0}\right|^{2}+\left|c_{h}^{0}\right|^{2}+1+\left\|\phi_{h}^{0}\right\|_{H^{1}(\Omega)}^{2} \\
& +2 \int_{\Omega} F\left(\phi_{h}^{0}\right)+k \sum_{l=0}^{n-1}\left(R_{1}\left\|\phi_{h}^{l}\right\|_{H^{1}}^{2}+R_{2} \frac{\beta^{2}}{\varepsilon^{4}}\left(\left|c_{h}^{l}\right|^{2}+1\right)\right) .
\end{aligned}
$$

Now, the discrete Gronwall lemma and (3.2) yield

$$
\begin{aligned}
\frac{2 C_{V} \beta}{l \varepsilon^{2}}\left|\theta_{h}^{n}\right|^{2}+\left|c_{h}^{n}\right|^{2}+1+\left\|\phi_{h}^{n}\right\|_{H^{1}(\Omega)}^{2}+2 \int_{\Omega} F\left(\phi_{h}^{n}\right) & \leq \mathrm{e}^{\left(R_{1}+\frac{\beta^{2}}{\varepsilon^{4}} R_{2}\right)(n-1) k}\left(\frac{2 C_{V} \beta}{l \varepsilon^{2}}\left|\theta_{h}^{0}\right|^{2}+\left|c_{h}^{0}\right|^{2}\right. \\
& \left.+1+\left\|\phi_{h}^{0}\right\|_{H^{1}(\Omega)}^{2}+2 \int_{\Omega} F\left(\phi_{h}^{0}\right)\right) \leq \mathrm{e}^{\left(R_{1}+\frac{\beta^{2}}{\varepsilon^{4}} R_{2}\right) T} C_{2} / \varepsilon^{2}:=C_{d} .
\end{aligned}
$$

Then, we find that hypothesis (6.4) is satisfied. Therefore, in view of Lemma 6.1, inequalities (6.5) and (6.6) hold.

Note that the stability estimates obtained in Lemma 6.2 are of order $O\left(\left(1 / \varepsilon^{2}\right) \mathrm{e}^{\left(\beta^{2} / \varepsilon^{4}\right)}\right)$ for the variable $\left(\phi, \frac{\sqrt{\beta}}{\varepsilon} \theta, c\right)$ as in the nonlinear scheme, but now an adequate constraint for $(h, k)$ small enough (depending exponentially on $1 / \varepsilon$ ) is necessary (recall that in the nonlinear scheme, only $\frac{\beta k}{\varepsilon^{2}}$ small enough was imposed).

To finish the proof of Theorem 1.4 it is necessary to prove the convergence of the linear scheme (6.1)-(6.3). But, as the argument for this is similar to that developed for the nonlinear scheme (2.1)-(2.3), it is left to the reader. 


\section{ERror estimates FOR THE NON-DEGENERATE CASE}

In this section we deal with the error analysis of both linear and nonlinear scheme. The presence of the truncation operator applied to the piecewise constant operator $P_{0}$ makes nonstandard this error analysis and this particular truncation is responsible of order $O(h)$ in error estimates, although higher-order finite elements were considered.

In order to be able to guarantee a sufficient regular solution of problem (1.1)-(1.3) we assume the nondegenerate case. For simplicity, we assume that $K_{1}$ and $K_{2}$ are positive constants, providing in particular standard Neumann boundary conditions in (1.2).

Let $\left\{\mathcal{T}_{h}\right\}, 0<h \leq 1$, be a regular, quasi-uniform family of subdivisions of a polyhedral domain $\Omega \subset \mathbb{R}^{m}$, $m=2$ or 3 , whose boundary $\Gamma$ is such that the problem

$$
-\Delta u+u=f \text { in } \Omega, \quad \frac{\partial u}{\partial \boldsymbol{n}}=0 \text { on } \Gamma
$$

holds the stability property $\|u\|_{H^{2}(\Omega)} \leq|f|$, for each $f \in L^{2}(\Omega)$. Recall that, both previous hypotheses are also assumed in Theorem 1.3 to prove the convergence.

Define the global error $e_{\phi}^{n}=\phi_{h}^{n}-\phi\left(t_{n}\right), e_{\theta}^{n}=\theta_{h}^{n}-\theta\left(t_{n}\right)$, and $e_{c}^{n}=c_{h}^{n}-c\left(t_{n}\right)$. These errors are decomposed into a discrete error $e_{\cdot, d}$, and an interpolation error $e_{\cdot, i}$ as follows

$$
\begin{aligned}
& e_{\phi, d}^{n}=\phi_{h}^{n}-P_{h}^{1} \phi\left(t_{n}\right), \quad e_{\phi, i}^{n}=P_{h}^{1} \phi\left(t_{n}\right)-\phi\left(t_{n}\right), \\
& e_{\theta, d}^{n}=\theta_{h}^{n}-P_{h}^{0} \theta\left(t_{n}\right), \quad e_{\theta, i}^{n}=P_{h}^{0} \theta\left(t_{n}\right)-\theta\left(t_{n}\right), \\
& e_{c, d}^{n}=c_{h}^{n}-P_{h}^{0} c\left(t_{n}\right), \quad e_{c, i}^{n}=P_{h}^{0} c\left(t_{n}\right)-c\left(t_{n}\right),
\end{aligned}
$$

where $P_{h}^{1}: H^{1} \rightarrow X_{h}$ is the $H^{1}$-projection operator defined as

$$
\left(\psi-P_{h}^{1} \psi, x_{h}\right)+\left(\nabla \psi-\nabla P_{h}^{1} \psi, \nabla x_{h}\right)=0 \quad \forall x_{h} \in X_{h}
$$

and $P_{h}^{0}: L^{2} \rightarrow X_{h}$ is the $L^{2}$-projection operator defined as

$$
\left(\psi-P_{h}^{0} \psi, x_{h}\right)=0 \quad \forall x_{h} \in X_{h}
$$

Finally, let us recall some approximation properties of $P_{h}^{1}$ and $P_{h}^{0}$ to be used later on (see [6], Prop. 1.134, p. 73):

$$
\begin{aligned}
\left\|\psi-P_{h}^{0} \psi\right\|_{H^{1}(\Omega)} & \leq C h\|\psi\|_{H^{2}(\Omega)}, \quad\left\|\psi-P_{h}^{1} \psi\right\|_{H^{1}(\Omega)} \leq C h\|\psi\|_{H^{2}(\Omega)}, \\
\left|\psi-P_{0} \psi\right| \leq C h\|\psi\|_{H^{1}(\Omega)}, & \left|\psi-P_{h}^{1} \psi\right| \leq C h\|\psi\|_{H^{1}(\Omega)}
\end{aligned}
$$

In particular, from the last inequality, one has

$$
k\left|\delta_{t} \psi\left(t_{n+1}\right)-\delta_{t} P_{h}^{1} \psi\left(t_{n+1}\right)\right|^{2} \leq C h^{2} \int_{t_{n}}^{t_{n+1}}\left\|\psi_{t}\right\|_{H^{1}(\Omega)}^{2},
$$

where we use $\delta_{t}$ to denote the discrete backward Euler time derivative, that is

$$
\delta_{t} \psi\left(t_{n+1}\right)=\frac{\psi\left(t_{n+1}\right)-\psi\left(t_{n}\right)}{k}
$$

Note that, for the $H^{1}$-interpolation error of $P_{h}^{0}$, a quasi-uniform family of finite elements must be assumed and, for the $L^{2}$-interpolation error of $P_{h}^{1}$, a duality argument is required where the elliptic $H^{2}$-regularity for the Elliptic-Neumann problem (7.1) is imposed. 
Throughout the section we assume a regular solution of (1.1)-(1.3). Concretely, one assumes

$$
\begin{aligned}
& \phi \in L^{\infty}\left(0, T ; W^{1, \infty}(\Omega)\right) \cap L^{2}\left(0, T ; H^{2}(\Omega)\right), \phi_{t} \in L^{2}\left(0, T ; H^{1}(\Omega), \phi_{t t} \in L^{2}\left(0, T ; L^{2}(\Omega)\right),\right. \\
& \theta \in L^{\infty}\left(0, T ; H^{1}(\Omega)\right) \cap L^{2}\left(0, T, H^{2}(\Omega)\right), \theta_{t} \in L^{2}\left(0, T ; L^{2}(\Omega)\right), \theta_{t t} \in L^{2}\left(0, T ; H^{1}(\Omega)^{\prime}\right), \\
& c \in L^{\infty}\left(0, T ; H^{1}(\Omega)\right) \cap L^{2}\left(0, T, H^{2}(\Omega)\right), c_{t} \in L^{2}\left(0, T ; L^{2}(\Omega)\right), c_{t t} \in L^{2}\left(0, T ; H^{1}(\Omega)^{\prime}\right) .
\end{aligned}
$$

\subsection{Error estimates for the nonlinear scheme}

We now state our error estimates for the fully discrete nonlinear scheme (2.1)-(2.3). If we compare the exact problem with the scheme and use the equality $a^{3}-b^{3}=(a-b)^{3}+3 a b(a-b)$, then the error equations are given by

$$
\begin{aligned}
& \left\{\begin{aligned}
\alpha & \left(\delta_{t} e_{\phi, d}^{n+1}, x_{h}\right)+\left(\nabla e_{\phi, d}^{n+1}, \nabla x_{h}\right)+\left(e_{\phi, d}^{n+1}, x_{h}\right)+\frac{1}{2 \varepsilon^{2}}\left(\left(e_{\phi, d}^{n+1}\right)^{3}, x_{h}\right) \\
= & -\alpha\left(\delta_{t} e_{\phi, i}^{n+1}, x_{h}\right)-\frac{1}{2 \varepsilon^{2}}\left(\left(e_{\phi, i}^{n+1}\right)^{3}, x_{h}\right)-\frac{3}{2 \varepsilon^{2}}\left(P_{h}^{1}\left(\phi\left(t_{n+1}\right)\right) \phi\left(t_{n+1}\right) e_{\phi, i}^{n+1}, x_{h}\right) \\
& -\frac{3}{2 \varepsilon^{2}}\left(\phi_{h}^{n+1} P_{h}^{1}\left(\phi\left(t_{n+1}\right)\right) e_{\phi, d}^{n+1}, x_{h}\right)+\frac{1}{2 \varepsilon^{2}}\left(e_{\phi, d}^{n}+e_{\phi, i}^{n}, x_{h}\right) \\
& +\left(e_{\phi, d}^{n}+e_{\phi, i}^{n}, x_{h}\right)+\frac{\beta}{\varepsilon^{2}}\left(e_{\theta, d}^{n}, x_{h}\right)-\frac{\beta}{\varepsilon^{2}}\left(\theta_{A}+\theta_{B}\right)\left(e_{c, d}^{n}, x_{h}\right)+\left(R_{\phi}^{n+1}, x_{h}\right),
\end{aligned}\right. \\
& \left\{\begin{array}{l}
C_{V}\left(\delta_{t} e_{\theta, d}^{n+1}, x_{h}\right)+K_{1}\left(\nabla e_{\theta, d}^{n+1}, \nabla x_{h}\right) \\
=-K_{1}\left(\nabla e_{\theta, i}^{n+1}, \nabla x_{h}\right)-\frac{l}{2}\left(\delta_{t} e_{\phi}^{n+1}, x_{h}\right)+\left(R_{\theta}^{n+1}, x_{h}\right),
\end{array}\right. \\
& \left\{\begin{array}{l}
\left(\delta_{t} e_{c, d}^{n+1}, x_{h}\right)+K_{2}\left(\nabla e_{c, d}^{n+1}, \nabla x_{h}\right)=-K_{2}\left(\nabla e_{c, i}^{n+1}, \nabla x_{h}\right) \\
-M K_{2}\left(\left[P_{0} c_{h}^{n}\right]_{T}\left(1-\left[P_{0} c_{h}^{n}\right]_{T}\right) \nabla e_{\phi}^{n}, \nabla x_{h}\right) \\
-M K_{2}\left(\left(1-\left[P_{0} c_{h}^{n}\right]_{T}\right) e_{c_{T}}^{n} \nabla \phi\left(t_{n}\right), \nabla x_{h}\right) \\
+M K_{2}\left(c\left(t_{n}\right) e_{c_{T}}^{n} \nabla \phi\left(t_{n}\right), \nabla x_{h}\right)+\left(R_{c}^{n+1}, x_{h}\right),
\end{array}\right.
\end{aligned}
$$

where $\delta_{t} e^{n+1}=\left(e^{n+1}-e^{n}\right) / k, e_{c_{T}}^{n}=\left[P_{0} c_{h}^{n}\right]_{T}-c\left(t_{n}\right)$ and

$$
\begin{aligned}
R_{\phi}^{n+1}= & \frac{\alpha}{k} \int_{t_{n}}^{t_{n+1}}\left(t_{n}-t\right) \phi_{t t}(t) \mathrm{d} t+\left(\frac{1}{2 \varepsilon^{2}}+1\right) \int_{t_{n}}^{t_{n+1}} \phi_{t}(t) \mathrm{d} t \\
& +\frac{\beta}{\varepsilon^{2}} \int_{t_{n}}^{t_{n+1}} \theta_{t}(t) \mathrm{d} t+\frac{\beta}{\varepsilon^{2}}\left(\theta_{A}+\theta_{B}\right) \int_{t_{n}}^{t_{n+1}} c_{t}(t) \mathrm{d} t \\
R_{\theta}^{n+1}= & \frac{C_{V}}{k} \int_{t_{n}}^{t_{n}}\left(t_{n}-t\right) \theta_{t t}(t) \mathrm{d} t-\frac{l}{2 k} \int_{t_{n}}^{t_{n+1}}\left(t_{n}-t\right) \phi_{t t}(t) \mathrm{d} t, \\
R_{c}^{n+1}= & \frac{1}{k} \int_{t_{n}}^{t_{n+1}}\left(t_{n}-t\right) c_{t t}(t) \mathrm{d} t+\nabla \cdot\left(\left(1-c\left(t_{n+1}\right)\right) c\left(t_{n+1}\right)\left(\int_{t_{n}}^{t_{n+1}} \nabla \phi_{t}(t)\right)\right. \\
+ & \left(1-\left(c\left(t_{n}\right)+c\left(t_{n+1}\right)\right)\left(\int_{t_{n}}^{t_{n+1}} c_{t}(t)\right) \nabla \phi\left(t_{n}\right)\right) .
\end{aligned}
$$

Theorem 7.1. Under the assumptions of Theorem 1.3, if the solution $(\phi, \theta, c)$ of (1.1)-(1.3) satisfies that $(\phi(t), \theta(t), c(t)) \in H^{2}(\Omega)^{3}$ for all $t \in[0, T]$ and the regularity given in (7.2), then the following error estimates hold for $k$ small enough:

$$
\max _{0 \leq n \leq N}\left(\left\|e_{\phi}^{n+1}\right\|_{H^{1}(\Omega)}^{2}+\left|e_{\theta}^{n+1}\right|^{2}+\left|e_{c}^{n+1}\right|^{2}\right)+k \sum_{n=0}^{N-1}\left(\left|\delta_{t} e_{\phi}^{n+1}\right|^{2}+\left|\nabla e_{\theta}^{n+1}\right|^{2}+\left|\nabla e_{c}^{n+1}\right|^{2}\right) \leq C\left(h^{2}+k^{2}\right),
$$


where the constant $C>0$ depends on the exact solution, but is independent of $(h, k)$.

Proof. Set $x_{h}=2 k \delta_{t} e_{\phi, d}^{n+1} \in X_{h}$ as a test function in (7.3), and using the equalities

$$
a^{3}(a-b)=\frac{1}{2} a^{2}\left(a^{2}-b^{2}+(a-b)^{2}\right)=\frac{1}{4}\left(a^{4}-b^{4}+\left(a^{2}-b^{2}\right)^{2}\right)+\frac{1}{2} a^{2}(a-b)^{2}
$$

for $a=e_{\phi}^{n+1}$ and $b=e_{\phi}^{n}$, we get

$$
\left\{\begin{array}{rl}
\alpha 2 & k\left|\delta_{t} e_{\phi, d}^{n+1}\right|^{2}+\left(\left\|e_{\phi, d}^{n+1}\right\|_{H^{1}(\Omega)}^{2}-\left\|e_{\phi, d}^{n}\right\|_{H^{1}(\Omega)}^{2}+\left\|e_{\phi, d}^{n+1}-e_{\phi, d}^{n}\right\|_{H^{1}(\Omega)}^{2}\right) \\
& +\frac{1}{4 \varepsilon^{2}}\left(\left\|e_{\phi, d}^{n+1}\right\|_{L^{4}(\Omega)}^{4}-\left\|e_{\phi, d}^{n}\right\|_{L^{4}(\Omega)}^{4}+\left|\left(e_{\phi, d}^{n+1}\right)^{2}-\left(e_{\phi, d}^{n}\right)^{2}\right|^{2}+2\left|\left(e_{\phi, d}^{n+1}\right)\left(e_{\phi, d}^{n+1}-e_{\phi, d}^{n}\right)\right|^{2}\right) \\
= & -\alpha 2 k\left(\delta_{t} e_{\phi, i}^{n+1}, \delta_{t} e_{\phi, d}^{n+1}\right)-\frac{1}{\varepsilon^{2}} k\left(\left(e_{\phi, i}^{n+1}\right)^{3}, \delta_{t} e_{\phi, d}^{n+1}\right) \\
& -\frac{3}{\varepsilon^{2}} k\left(P_{h}^{1}\left(\phi\left(t_{n+1}\right)\right) \phi\left(t_{n+1}\right) e_{\phi, i}^{n+1}, \delta_{t} e_{\phi, d}^{n+1}\right)-\frac{3}{\varepsilon^{2}} k\left(\phi_{h}^{n+1} P_{h}^{1}\left(\phi\left(t_{n+1}\right)\right) e_{\phi, d}^{n+1}, \delta_{t} e_{\phi, d}^{n+1}\right) \\
& +\frac{1}{\varepsilon^{2}} k\left(e_{\phi, d}^{n}+e_{\phi, i}^{n}, \delta_{t} e_{\phi, d}^{n+1}\right)+2 k\left(e_{\phi, d}^{n}+e_{\phi, i}^{n}, \delta_{t} e_{\phi, d}^{n+1}\right) \\
& +2 \frac{\beta}{\varepsilon^{2}} k\left(e_{\theta, d}^{n}, \delta_{t} e_{\phi, d}^{n+1}\right)-2 \frac{\beta}{\varepsilon^{2}}\left(\theta_{A}+\theta_{B}\right) k\left(e_{c, d}^{n}, \delta_{t} e_{\phi, d}^{n+1}\right)+2 k\left(R_{\phi}^{n+1}, \delta_{t} e_{\phi, d}^{n+1}\right):=\sum_{i=1}^{9} I_{i} .
\end{array}\right.
$$

Now, we must bound each term on the right-hand side of (7.8). We just focus on the terms $I_{2}, I_{3}$ and $I_{4}$ :

$$
\begin{aligned}
I_{2} & \leq C_{\lambda} \frac{1}{\varepsilon^{4} \alpha} k\left\|e_{\phi, i}^{n+1}\right\|_{L^{6}(\Omega)}^{6}+\lambda k \alpha\left|\delta_{t} e_{\phi, d}^{n+1}\right|^{2} \leq C_{\lambda} \frac{1}{\varepsilon^{4} \alpha} k h^{6}\left\|\phi\left(t_{n+1}\right)\right\|_{H^{2}(\Omega)}^{6}+\lambda k \alpha\left|\delta_{t} e_{\phi, d}^{n+1}\right|^{2}, \\
I_{3} & \leq \frac{3}{\varepsilon^{2}} k\left\|P_{h}^{1}\left(\phi\left(t_{n+1}\right)\right)\right\|_{L^{6}(\Omega)}\left\|\phi\left(t_{n+1}\right)\right\|_{L^{6}(\Omega)}\left\|e_{\phi, i}^{n+1}\right\|_{L^{6}(\Omega)}\left|\delta_{t} e_{\phi, d}^{n+1}\right| \\
& \leq C_{\lambda} \frac{1}{\varepsilon^{4} \alpha} k\left\|\phi\left(t_{n+1}\right)\right\|_{H^{1}(\Omega)}^{4}\left\|e_{\phi, i}^{n+1}\right\|_{H^{1}(\Omega)}^{2}+\lambda k \alpha\left|\delta_{t} e_{\phi, d}^{n+1}\right|^{2} \\
& \leq C_{\lambda} \frac{1}{\varepsilon^{4} \alpha} k h^{2}\left\|\phi\left(t_{n+1}\right)\right\|_{H^{1}(\Omega)}^{4}\left\|\phi\left(t_{n+1}\right)\right\|_{H^{2}(\Omega)}^{2}+\lambda k \alpha\left|\delta_{t} e_{\phi, d}^{n+1}\right|^{2}, \\
I_{4} & \leq \frac{3}{\varepsilon^{2}} k\left\|\phi_{h}^{n+1}\right\|_{L^{6}(\Omega)}\left\|P_{h}^{1}\left(\phi\left(t_{n+1}\right)\right)\right\|_{L^{6}(\Omega)}\left\|e_{\phi, d}^{n+1}\right\|_{L^{6}(\Omega)}\left|\delta_{t} e_{\phi, d}^{n+1}\right| \\
& \leq C_{\lambda} \frac{1}{\varepsilon^{4} \alpha} k\left\|\phi\left(t_{n+1}\right)\right\|_{H^{1}(\Omega)}^{2}\left\|e_{\phi, d}^{n+1}\right\|_{H^{1}(\Omega)}^{2}+\lambda k \alpha\left|\delta_{t} e_{\phi, d}^{n+1}\right|^{2} .
\end{aligned}
$$

In the last line, the stability estimate $\left\|\phi_{h}^{n+1}\right\|_{L^{6}(\Omega)} \leq C$ given in Lemma 3.1 has been applied. Note that the previous inequalities hold for any $\lambda>0$ and $C_{\lambda}>0$ are different constants of order $O(1 / \lambda)$. Finally, the constants $C_{\lambda}$ bounding $I_{2}$ and $I_{3}$ are independent of $\varepsilon$, but the constant $C_{\lambda}$ related to $I_{4}$ depends on $\varepsilon$ via the bound of Lemma 3.1 .

The remainder of the terms can be bounded more easily. Thus, by choosing $\lambda$ small enough, we get

$$
\left\{\begin{array}{c}
\alpha k\left|\delta_{t} e_{\phi, d}^{n+1}\right|^{2}+\left(\left\|e_{\phi, d}^{n+1}\right\|_{H^{1}(\Omega)}^{2}-\left\|e_{\phi, d}^{n}\right\|_{H^{1}(\Omega)}^{2}+\left\|e_{\phi, d}^{n+1}-e_{\phi, d}^{n}\right\|_{H^{1}(\Omega)}^{2}\right) \\
\quad+\frac{1}{4 \varepsilon^{2}}\left(\left\|e_{\phi, d}^{n+1}\right\|_{L^{4}(\Omega)}^{4}-\left\|e_{\phi, d}^{n}\right\|_{L^{4}(\Omega)}^{4}+\left|\left(e_{\phi, d}^{n+1}\right)^{2}-\left(e_{\phi, d}^{n}\right)^{2}\right|^{2}+2\left|e_{\phi, d}^{n+1}\left(e_{\phi, d}^{n+1}-e_{\phi, d}^{n}\right)\right|^{2}\right) \\
\leq C k h^{2}\left(h^{4}\left\|\phi\left(t_{n+1}\right)\right\|_{H^{2}(\Omega)}^{6}+\left\|\phi\left(t_{n+1}\right)\right\|_{H^{1}(\Omega)}^{4}\left\|\phi\left(t_{n+1}\right)\right\|_{H^{2}(\Omega)}^{2}+\left\|\phi\left(t_{n}\right)\right\|_{H^{1}(\Omega)}^{2}\right) \\
\quad+C k \frac{1}{\varepsilon^{4} \alpha}\left(\beta^{2}\left|e_{c, d}^{n}\right|^{2}+\beta^{2} C_{V}\left|e_{\theta, d}^{n}\right|^{2}+\left\|e_{\phi, d}^{n}\right\|_{H^{1}(\Omega)}^{2}+\left\|\phi\left(t_{n+1}\right)\right\|_{H^{1}(\Omega)}^{2}\left\|e_{\phi, d}^{n+1}\right\|_{H^{1}(\Omega)}^{2}\right) \\
+C h^{2} \int_{t_{n}}^{t_{n+1}}\left\|\phi_{t}(s)\right\|_{H^{1}(\Omega)}^{2}+C k^{2} \int_{t_{n}}^{t_{n+1}}\left(\left|\phi_{t}(s)\right|^{2}+\left|\theta_{t}(s)\right|^{2}+\left|c_{t}(s)\right|^{2}+\left|\phi_{t t}(s)\right|^{2}\right) \mathrm{d} s
\end{array}\right.
$$

where $C>0$ are different constants independent of $(h, k)$ and independent of the exact solution $(\phi, \theta, c)$. 
We now test (7.4) with $2 k e_{\theta, d}^{n+1}$ and bound the right-hand side

$$
\left\{\begin{aligned}
& C_{V}\left(\left|e_{\theta, d}^{n+1}\right|^{2}-\left|e_{\theta, d}^{n}\right|^{2}+\left|e_{\theta, d}^{n+1}-e_{\theta, d}^{n}\right|^{2}\right)+2 K_{1} k\left|\nabla e_{\theta, d}^{n+1}\right|^{2} \\
= & -2 K_{1} k\left(\nabla e_{\theta, i}^{n+1}, \nabla e_{\theta, d}^{n+1}\right)-l k\left(\delta_{t} e_{\phi, d}^{n+1}+\delta_{t} e_{\phi, i}^{n+1}, e_{\theta, d}^{n+1}\right)+2 k\left(R_{\theta}^{n+1}, e_{\theta, d}^{n+1}\right) \\
\leq & C k h^{2}\left\|\theta\left(t_{n+1}\right)\right\|_{H^{2}(\Omega)}^{2+1}+K_{1} k\left|\nabla e_{\theta, d}^{n+1}\right|^{2}+C h^{2} \int_{t_{n}}^{t_{n+1}}\left\|\phi_{t}(s)\right\|_{H^{1}(\Omega)}^{2} \mathrm{~d} s \\
& +\frac{\alpha}{2} k\left|\delta_{t} e_{\phi, d}^{n+1}\right|^{2}+C C_{V} k\left|e_{\theta, d}^{n+1}\right|^{2}+C k^{2} \int_{t_{n}}^{t_{n+1}}\left(\left\|\theta_{t t}(s)\right\|_{H^{1}(\Omega)^{\prime}}^{2}+\left\|\phi_{t t}(s)\right\|_{H^{1}(\Omega)^{\prime}}^{2}\right) \mathrm{d} s .
\end{aligned}\right.
$$

Let us now take $x_{h}=2 k e_{c, d}^{n+1}$ as a test function into (7.5),

$$
\left\{\begin{aligned}
\left|e_{c, d}^{n+1}\right|^{2}-\left|e_{c, d}^{n}\right|^{2}+\left|e_{c, d}^{n+1}-e_{c, d}^{n}\right|^{2}+2 K_{2} k\left|\nabla e_{c, d}^{n+1}\right|^{2} \\
=-2 K_{2} k\left(\nabla e_{c, i}^{n+1}, \nabla e_{c, d}^{n+1}\right)-2 M K_{2} k\left(\left[P_{0} c_{h}^{n}\right]_{T}\left(1-\left[P_{0} c_{h}^{n}\right]_{T}\right) \nabla\left(e_{\phi, d}^{n}+e_{\phi, i}^{n}\right), \nabla e_{c, d}^{n+1}\right) \\
\quad-2 M K_{2} k\left(\left(1-\left[P_{0} c_{h}^{n}\right]_{T}\right) e_{c T}^{n} \nabla \phi\left(t_{n}\right), \nabla e_{c, d}^{n+1}\right) \\
\quad+2 M K_{2} k\left(c\left(t_{n}\right) e_{c_{T}}^{n} \nabla \phi\left(t_{n}\right), \nabla e_{c, d}^{n+1}\right)+2 k\left(R_{c}^{n+1}, e_{c, d}^{n+1}\right) .
\end{aligned}\right.
$$

We firstly bound the truncated error

$$
\begin{aligned}
\left|e_{c_{T}}^{n}\right|^{2} & =\left|\left[P_{0} c_{h}^{n}\right]_{T}-P_{0} c\left(t_{n}\right)+P_{0} c\left(t_{n}\right)-c\left(t_{n}\right)\right|^{2} \\
& \leq C\left(\left|\left[P_{0} c_{h}^{n}\right]_{T}-P_{0} c\left(t_{n}\right)\right|^{2}+\left|P_{0} c\left(t_{n}\right)-c\left(t_{n}\right)\right|^{2}\right) \\
& \leq C\left(\left|P_{0} c_{h}^{n}-P_{0} c\left(t_{n}\right)\right|^{2}+h^{2}\left|\nabla c\left(t_{n}\right)\right|^{2}\right) \\
& \leq C\left(\left|e_{c, d}^{n}\right|^{2}+\left|e_{c, i}^{n}\right|^{2}+h^{2}\left|\nabla c\left(t_{n}\right)\right|^{2}\right) \\
& \leq C\left(\left|e_{c, d}^{n}\right|^{2}+h^{2}\left\|c\left(t_{n}\right)\right\|_{H^{1}(\Omega)}^{2}\right)
\end{aligned}
$$

where in the last line we have used the stability property $\left|P_{0} \psi\right| \leq|\psi|$. Note that the interpolation error $\left|P_{0} c\left(t_{n}\right)-c\left(t_{n}\right)\right|$ appearing in (7.11) is only of order $O(h)$, independent of the finite element approximation.

By virtue of (7.11), we bound

$$
\left\{\begin{array}{l}
\left|e_{c, d}^{n+1}\right|^{2}-\left|e_{c, d}^{n}\right|^{2}+\left|e_{c, d}^{n+1}-e_{c, d}^{n}\right|^{2}+K_{2} k\left|\nabla e_{c, d}^{n+1}\right|^{2} \\
\leq C k h^{2}\left\|c\left(t_{n+1}\right)\right\|_{H^{2}(\Omega)}^{2}+C k\left|\nabla e_{\phi, d}^{n}\right|^{2}+C k h^{2}\left\|\nabla \phi\left(t_{n}\right)\right\|_{L^{\infty}(\Omega)}^{2}\left\|c\left(t_{n}\right)\right\|_{H^{1}(\Omega)}^{2} \\
\quad+C k h^{2}\left\|\phi\left(t_{n}\right)\right\|_{H^{2}(\Omega)}^{2}+C k\left\|\nabla \phi\left(t_{n}\right)\right\|_{L^{\infty}(\Omega)}^{2}\left|e_{c, d}^{n}\right|^{2} \\
\quad+C k^{2} \int_{t_{n}}^{t_{n+1}}\left|\nabla \phi_{t}(s)\right|^{2} \mathrm{~d} s \\
\quad+C k^{2}\left\|\nabla \phi\left(t_{n+1}\right)\right\|_{L^{\infty}}^{2} \int_{t_{n}}^{t_{n+1}}\left|c_{t}(s)\right|^{2} \mathrm{~d} s+C k^{2} \int_{t_{n}}^{t_{n+1}}\left\|c_{t t}(s)\right\|_{H^{1}(\Omega)^{\prime}}^{2} \mathrm{~d} s .
\end{array}\right.
$$

Again, $C>0$ are different constants independent of $(h, k)$ and independent of the exact solution $(\phi, \theta, c)$. 
By adding (7.9), (7.10) and (7.12) and applying the generalized discrete Gronwall lemma, we establish the following estimate for all $n<N$ and for $k$ small enough:

$$
\begin{aligned}
\left\|e_{\phi, d}^{n+1}\right\|_{H^{1}(\Omega)}^{2}+\frac{1}{4 \varepsilon^{2}}\left\|e_{\phi, d}^{n+1}\right\|_{L^{4}(\Omega)}^{4}+ & C_{V}\left|e_{\theta, d}^{n+1}\right|^{2}+\left|e_{c, d}^{n+1}\right|^{2}+\alpha k \sum_{l=0}^{n}\left|\delta_{t} e_{\phi, d}^{l+1}\right|^{2} \\
& +K_{1} k \sum_{l=0}^{n}\left|\nabla e_{\theta, d}^{l+1}\right|^{2}+K_{2} k \sum_{l=0}^{n}\left|\nabla e_{c, d}^{l+1}\right|^{2} \leq \exp \left(C_{1} T\right)\left(C_{2} h^{2}+C_{3} k^{2}\right),
\end{aligned}
$$

where

$$
\begin{gathered}
C_{1}=\frac{C\left(1+\|\phi\|_{L^{2}\left(0, T ; W^{1, \infty}(\Omega)\right)}^{2}\right)}{1-C k \max \left\{1,\|\phi\|_{L^{\infty}\left(0, T ; H^{1}(\Omega)\right)}^{2}\right\}} \\
C_{2}=C\left(\|\phi\|_{L^{\infty}\left(0, T ; H^{1}(\Omega)\right)}^{4}\|\phi\|_{L^{2}\left(0, T ; H^{2}(\Omega)\right)}^{2}+\|\phi\|_{L^{2}\left(0, T ; H^{2}(\Omega)\right)}^{2}\right. \\
+\left\|\phi_{t}^{2}\right\|_{L^{2}\left(0, T ; H^{1}(\Omega)\right)}^{2}+\|\theta\|_{L^{2}\left(0, T ; H^{2}(\Omega)\right)}^{2} \\
\left.+\|\phi\|_{L^{2}\left(0, T ; W^{1, \infty}(\Omega)\right)}^{2}\|c\|_{L^{\infty}\left(0, T ; H^{1}(\Omega)\right)}^{2}+\|c\|_{L^{2}\left(0, T ; H^{2}(\Omega)\right)}^{2}\right),
\end{gathered}
$$

and

$$
\begin{aligned}
C_{3}= & C\left(\left\|\phi_{t}\right\|_{L^{2}\left(0, T ; L^{2}(\Omega)\right)}^{2}+\left\|\theta_{t}\right\|_{L^{2}\left(0, T ; L^{2}(\Omega)\right)}^{2}+\left\|c_{t}\right\|_{L^{2}\left(0, T ; L^{2}(\Omega)\right)}^{2}+\left\|\phi_{t t}\right\|_{L^{2}\left(0, T ; L^{2}(\Omega)\right)}^{2}\right. \\
& +\left\|\theta_{t t}\right\|_{L^{2}\left(0 ; T ; H^{1}(\Omega)^{\prime}\right)}^{2}+\left\|\phi_{t t}\right\|_{L^{2}\left(0, T ; H^{1}(\Omega)^{\prime}\right)}^{2}+\left\|\phi_{t}\right\|_{L^{2}\left(0, T ; H^{1}(\Omega)\right)}^{2} \\
& \left.+\|\phi\|_{L^{\infty}\left(0, T ; W^{1, \infty}(\Omega)\right)}^{2}\left\|c_{t}\right\|_{L^{2}\left(0, T ; L^{2}(\Omega)\right)}^{2} \mathrm{~d} s+\left\|c_{t t}\right\|_{L^{2}\left(0, T ; H^{1}(\Omega)^{\prime}\right)}^{2}\right)
\end{aligned}
$$

with $C>0$ independent of $(h, k)$ and independent of the exact solution $(\phi, \theta, c)$.

The same estimates are obtained for the total errors by using the interpolation errors; hence (7.7) can be deduced.

Remark 7.2. Observe that, to obtain error estimates in the previous theorem, the monotony property (analogous to (3.8))

$$
\frac{1}{2 \varepsilon^{2}}\left(\left(e_{\phi, d}^{n+1}\right)^{3}-e_{\phi, d}^{n}\right)\left(e_{\phi, d}^{n+1}-e_{\phi, d}^{n}\right) \geq F\left(e_{\phi, d}^{n+1}\right)-F\left(e_{\phi, d}^{n}\right)
$$

is not used, because the corresponding initial term is

$$
\int_{\Omega} F\left(e_{\phi, d}^{0}\right)=\frac{1}{8 \varepsilon^{2}} \int_{\Omega}\left(\left(e_{\phi, d}^{0}\right)^{2}-1\right)^{2}=O\left(\frac{1}{\varepsilon^{2}}\right) .
$$

Therefore, by using this property, the error estimates of order $O(k+h)$ does not hold, because in this case the final bound remains of order $O\left(k+h+1 / \varepsilon^{2}\right)$.

\subsection{Error estimates for the linear scheme}

As the derivation of the error equations for $\theta_{h}^{n+1}$ and $c_{h}^{n+1}$ is exactly the same as in the previous nonlinear scheme, we only treat in this section the error equation for $\phi_{h}^{n+1}$, which is given by

$$
\left\{\begin{aligned}
\alpha & \left(\delta_{t} e_{\phi, d}^{n+1}, x_{h}\right)+\left(\nabla e_{\phi, d}^{n+1}, \nabla x_{h}\right)+\left(e_{\phi, d}^{n+1}, x_{h}\right)+\frac{1}{2 \varepsilon^{2}}\left(\left(e_{\phi, d}^{n}\right)^{3}, x_{h}\right) \\
= & -\alpha\left(\delta_{t} e_{\phi, i}^{n+1}, x_{h}\right)-\frac{1}{2 \varepsilon^{2}}\left(\left(e_{\phi, i}^{n}\right)^{3}, x_{h}\right)-\frac{3}{2 \varepsilon^{2}}\left(P_{h}^{1}\left(\phi\left(t_{n}\right)\right) \phi\left(t_{n}\right) e_{\phi, i}^{n}, x_{h}\right) \\
& -\frac{3}{2 \varepsilon^{2}}\left(\phi_{h}^{n} P_{h}^{1}\left(\phi\left(t_{n}\right)\right) e_{\phi, d}^{n}, x_{h}\right)+\frac{1}{2 \varepsilon^{2}}\left(e_{\phi, d}^{n}+e_{\phi, i}^{n}, x_{h}\right) \\
& +\left(e_{\phi, d}^{n}+e_{\phi, i}^{n}, x_{h}\right)+\frac{\beta}{\varepsilon^{2}}\left(e_{\theta, d}^{n}-\left(\theta_{A}+\theta_{B}\right) e_{c, d}^{n}, x_{h}\right)+\left(R_{\phi}^{n+1}+S_{\phi}^{n+1}, x_{h}\right)
\end{aligned}\right.
$$


where $R_{\phi}^{n+1}$ is as above and

$$
S_{\phi}^{n+1}=-\frac{1}{2 \varepsilon^{2}}\left(\phi\left(t_{n+1}\right)^{3}-\phi\left(t_{n}\right)^{3}\right)=-\frac{3}{2 \varepsilon^{2}} \int_{t_{n}}^{t_{n+1}} \phi(s)^{2} \phi_{t}(s) \mathrm{d} s .
$$

Again, we take $x_{h}=2 k \delta_{t} e_{\phi, d}^{n+1}$ as a test function in (7.14), and all computations work as for the nonlinear scheme, except the explicit term. Now, by using the equalities

$$
\begin{aligned}
b^{3}(a-b) & =\left[b a^{2}+b\left(b^{2}-a^{2}\right)\right](a-b)=\frac{1}{2} a^{2}\left(a^{2}-b^{2}-(a-b)^{2}\right)+b\left(b^{2}-a^{2}\right)(a-b) \\
& =\frac{1}{4}\left(a^{4}-b^{4}+\left(a^{2}-b^{2}\right)^{2}\right)-\frac{1}{2} a^{2}(a-b)^{2}+b\left(b^{2}-a^{2}\right)(a-b)
\end{aligned}
$$

for $a=e_{\phi, d}^{n+1}$ and $b=e_{\phi, d}^{n}$, we get

$$
\begin{aligned}
\frac{k}{\varepsilon^{2}}\left(\left(e_{\phi, d}^{n}\right)^{3}, \delta_{t} e_{\phi, d}^{n+1}\right)= & \frac{1}{4 \varepsilon^{2}}\left(\left\|e_{\phi, d}^{n+1}\right\|_{L^{4}(\Omega)}^{4}-\left\|e_{\phi, d}^{n}\right\|_{L^{4}(\Omega)}^{4}+\left|\left(e_{\phi, d}^{n+1}\right)^{2}-\left(e_{\phi, d}^{n}\right)^{2}\right|^{2}\right) \\
& -\frac{1}{2 \varepsilon^{2}} \mid e_{\phi, d}^{n+1}\left(e_{\phi, d}^{n+1}-\left.e_{\phi, d}^{n}\right|^{2}+\frac{k}{\varepsilon^{2}}\left(e_{\phi, d}^{n}\left[\left(e_{\phi, d}^{n}\right)^{2}-\left(e_{\phi, d}^{n+1}\right)^{2}\right], \delta_{t} e_{\phi, d}^{n+1}\right) .\right.
\end{aligned}
$$

Applying the inverse inequality $\left\|e_{\phi, d}^{n+1}\right\|_{L^{\infty}(\Omega)}^{2} \leq(C / h)\left\|e_{\phi, d}^{n+1}\right\|_{H^{1}(\Omega)}^{2}$ and the stability estimates of the (linear) scheme $\left\|\phi_{h}^{n}\right\|_{H^{1}(\Omega)} \leq C$ given in Lemma 6.2 , we can bound the last two terms as follows

$$
\begin{aligned}
\frac{1}{2 \varepsilon^{2}}\left|e_{\phi, d}^{n+1}\left(e_{\phi, d}^{n+1}-e_{\phi, d}^{n}\right)\right|^{2} & \leq \frac{1}{2 \varepsilon^{2}} k^{2}\left\|e_{\phi, d}^{n+1}\right\|_{L^{\infty}(\Omega)}^{2}\left|\delta_{t} e_{\phi, d}^{n+1}\right|^{2} \\
& \leq C_{\varepsilon} \frac{k^{2}}{h}\left\|e_{\phi, d}^{n+1}\right\|_{H^{1}(\Omega)}^{2}\left|\delta_{t} e_{\phi, d}^{n+1}\right|^{2} \leq C_{\varepsilon} \frac{k}{h} k\left|\delta_{t} e_{\phi, d}^{n+1}\right|^{2}, \\
\frac{k}{\varepsilon^{2}}\left(e_{\phi, d}^{n}\left[\left(e_{\phi, d}^{n}\right)^{2}-\left(e_{\phi, d}^{n+1}\right)^{2}\right], \delta_{t} e_{\phi, d}^{n+1}\right) & \leq C_{\varepsilon} k^{2}\left\|e_{\phi, d}^{n}\right\|_{L^{\infty}(\Omega)}^{2}\left|\delta_{t} e_{\phi, d}^{n+1}\right|^{2}+\frac{1}{8 \varepsilon^{2}}\left|\left(e_{\phi, d}^{n}\right)^{2}-\left(e_{\phi, d}^{n+1}\right)^{2}\right|^{2} \\
& \leq C_{\varepsilon} \frac{k}{h} k\left|\delta_{t} e_{\phi, d}^{n+1}\right|^{2}+\frac{1}{8 \varepsilon^{2}}\left|\left(e_{\phi, d}^{n}\right)^{2}-\left(e_{\phi, d}^{n+1}\right)^{2}\right|^{2} .
\end{aligned}
$$

Therefore, proceeding as in the nonlinear scheme, we get the same error estimates (7.7) by using the constraint $(S) k / h \rightarrow 0$. Notice that this time we need not make an induction argument thanks to the stability estimates provided by Lemma 6.2 .

Remark 7.3. All the above error estimates hold for the modified scheme where the term $c_{h}^{n}$ in $(2.1)$ (respectively, in (6.1)) is replaced by $\left[P_{0} c_{h}^{n}\right]_{T}$. To this end, we have to take into account estimate (7.11). We have already seen in Remark 3.2 that this type of more truncated schemes has stability constants depending on $1 / \varepsilon$ only of polynomial form.

Acknowledgements. We would like to thank to the anonymous referees for suggesting many improvements and for proposing the study of the error estimates.

\section{REFERENCES}

[1] J.L. Boldrini and G. Planas, Weak solutions of a phase-field model for phase change of an alloy with thermal properties. Math. Methods Appl. Sci. 25 (2002) 1177-1193.

[2] J.L. Boldrini and C. Vaz, A semidiscretization scheme for a phase-field type model for solidification. Port. Math. (N.S.) 63 (2006) 261-292.

[3] S. Brenner and L.R. Scott, The Mathematical Theory of Finite Element Methods, Texts in Applied Mathathematics 15. Springer-Verlag, Berlin (1994). 
[4] E. Burman, D. Kessler and J. Rappaz, Convergence of the finite element method applied to an anisotropic phase-field model. Ann. Math. Blaise Pascal 11 (2004) 67-94.

[5] G. Caginalp and W. Xie, Phase-field and sharp-interface alloy models. Phys. Rev. E 48 (1993) 1897-1909.

[6] A. Ern and J.L.Guermond, Theory and practice of finite elements, Applied Mathematical Sciences 159. Springer, New York (2004).

[7] X. Feng and A. Prohl, Analysis of a fully discrete finite element method for the phase field model and approximation of its sharp interface limits. Math. Comp. 73 (2004) 541-567.

[8] F. Guillén-González and J.V. Gutiérrez-Santacreu, Unconditional stability and convergence of a fully discrete scheme for $2 D$ viscous fluids models with mass diffusion. Math. Comp. 77 (2008) 1495-1524 (electronic).

[9] O. Kavian, Introduction à la Théorie des Points Critiques, Mathématiques et Applications 13. Springer, Berlin (1993).

[10] D. Kessler and J.F. Scheid, A priori error estimates of a finite-element method for an isothermal phase-field model related to the solidification process of a binary alloy. IMA J. Numer. Anal. 22 (2002) 281-305.

[11] R. Rannacher and R. Scott, Some optimal error estimates for piecewise linear finite element approximations. Math. Comp. 38 (1982) 437-445.

[12] J.F. Scheid, Global solutions to a degenerate solutal phase field model for the solidification of a binary alloy. Nonlinear Anal. 5 (2004) 207-217.

[13] J. Simon, Compact sets in the Space $L^{p}(0, T ; B)$. Ann. Mat. Pura Appl. 146 (1987) 65-97. 